\title{
Validation of Vehicle Driving Simulator from Perspective of Velocity and Trajectory Based Driving Behavior under Curve Conditions
}

\author{
Liang Chen $\left.{ }^{1} \mathbb{(}\right)$, Jiming Xie ${ }^{1}$, Simin $W_{u}{ }^{1}$, Fengxiang Guo ${ }^{1, *}$, Zheng Chen $\left.{ }^{1} \mathbb{(}\right)$ and Wenqi Tan ${ }^{2}$ \\ 1 Faculty of Transportation Engineering, Kunming University of Science and Technology, \\ Kunming 650504, China; cl@kust.edu.cn (L.C.); xiejiming@stu.kust.edu.cn (J.X.); \\ wusm@stu.kust.edu.cn (S.W.); chen@kust.edu.cn (Z.C.) \\ 2 College of Information and Smart Electromechanical Engineering, Xiamen Huaxia University, \\ Xiamen 361024, China; tanwq@hxxy.edu.cn \\ * Correspondence: guofengxiang@kust.edu.cn; Tel.: +86-871-65920131
}

Citation: Chen, L.; Xie, J.; Wu, S.; Guo, F.; Chen, Z.; Tan, W. Validation of Vehicle Driving Simulator from Perspective of Velocity and Trajectory Based Driving Behavior under Curve Conditions. Energies 2021, 14, 8429. https://doi.org/10.3390/en14248429

Academic Editors: Guzek Marek, Rafał Jurecki and Wojciech Wach

Received: 16 October 2021

Accepted: 8 December 2021

Published: 14 December 2021

Publisher's Note: MDPI stays neutral with regard to jurisdictional claims in published maps and institutional affiliations.

Copyright: (c) 2021 by the authors. Licensee MDPI, Basel, Switzerland. This article is an open access article distributed under the terms and conditions of the Creative Commons Attribution (CC BY) license (https:// creativecommons.org/licenses/by/ $4.0 /)$.

\begin{abstract}
With their advantages of high experimental safety, convenient setting of scenes, and easy extraction of control parameters, driving simulators play an increasingly important role in scientific research, such as in road traffic environment safety evaluation and driving behavior characteristics research. Meanwhile, the demand for the validation of driving simulators is increasing as its applications are promoted. In order to validate a driving simulator in a complex environment, curve road conditions with different radii are considered as experimental evaluation scenarios. To attain this, this paper analyzes the reliability and accuracy of the experimental vehicle speed of a driving simulator. Then, qualitative and quantitative analysis of the lateral deviation of the vehicle trajectory is carried out, applying the cosine similarity method. Furthermore, a data-driven method was adopted which takes the longitudinal displacement, lateral displacement, vehicle speed and steering wheel angle of the vehicle as inputs and the lateral offset as the output. Thus, a curve trajectory planning model, a more comprehensive and human-like operation, is established. Based on directional long short-term memory (Bi-LSTM) and a recurrent neural network (RNN), a multiple Bi-LSTM (Mul-Bi-LSTM) is proposed. The prediction performance of LSTM, MLP model and Mul-Bi-LSTM are compared in detail on the validation set and testing set. The results show that the Mul-Bi-LSTM model can generate a trajectory which is very similar to the driver's curve driving and have a preferable generalization performance. Therefore, this method can solve problems which cannot be realized in real complex scenes in the simulator validation. Selecting the trajectory as the validation parameter can more comprehensively and intuitively reflect the simulator's curve driving state. Using a speed model and trajectory model instead of a real car experiment can improve the efficiency of simulator validation and lay a foundation for the standardization of simulator validation.
\end{abstract}

Keywords: vehicle driving simulator; curve driving behavior; validation; multiple bi-directional long short-term memory (Mul-Bi-LSTM)

\section{Introduction}

In recent years, with the development of virtual reality technology towards practical applications, vehicle driving simulators have gradually become an important development direction in simulating the "people-vehicle-road-environment" system of road traffic research [1,2]. A vehicle driving simulator is a typical representative of a human-vehicleroad-environment simulation system. It exploits electronic computer images, with the support of electronic control and other technical support, to conduct manned simulation and research, including vehicle driving behavior, dynamic performance and traffic systems. The driving simulator has the distinguishing features of high safety, anticipated reproducibility, strong exploitability and low cost [3-6]. It can provide a safe environment 
for driving research and conveniently formulate a research approach related to driving behavior strategies. In particular, the experimental conditions feature a wide range and can be adjusted, and are also easy to change. Furthermore, the experimental data can be processed online, and classified and stored by a computer, which provides great convenience for the consequent statistical analysis $[7,8]$. Until now, driving simulators have been widely applied in research on intelligent control of vehicles, road traffic facilities and intelligent transportation systems $[9,10]$. They have become an effective auxiliary means and research tool for studying human efficiency, civil engineering, traffic engineering, psychology and related fields [11-15]. However, a vehicle driving simulator can only partially simulate an extremely complex transportation system. Meanwhile, each vehicle driving simulator experiment [16-18] involves effectiveness of experimental design, the rationality of content selection and representativeness of the selection of the sample (subjects), as well as the accuracy of the scene design, all of which may affect the experimental results, and even make the simulation results inconsistent with real situations. Therefore, how to validate the performance of a vehicle driving simulator deserves to be further investigated.

Until now, the simulator validation method has changed with the development of techniques and the extension of its applications. In the initial stage, the validation of simulators focuses on the physical characteristics of the vehicle. In the intermediate stage, the validation of simulators focuses on virtual images and vehicle kinematics. At present, the validation of simulators focuses on the characteristics of human-computer interaction, which is mainly due to the leading of cutting-edge technologies, such as humancomputer driving intelligent vehicle and advanced driver assistance systems (ADAS). Bham et al. [7] combined the objective evaluation and subjective evaluation to validate the driving simulator by comparing the simulation results with the real operation data collected by the global positioning system (GPS) along the highway and the video records of specific locations in the specific working area. Meuleners et al. [8] recruited 47 testers to participate in experiments and suggested they drive along a selected route on the real road and a virtual road in the driving simulator. Then, the driving simulator was calibrated by evaluating the behaviors of the driver in observing the left, right, front-viewing behavior, the speed of the intersection, the speed of following the vehicle and the observance of traffic lights and stop signs under two conditions. John A. Groeger et al. [19] compared the drivers' behavior characteristics of simulator driving and the real car driving under different curvature curves based on real vehicle experiment and two simulator simulation experiments. Through the analysis of vehicle speeds, position steering behavior and other parameters on curved road sections with different radii, the correlation of these parameters between the real vehicle and the simulator is attained to complete the effectiveness calibration of the driving simulator. Yanning Zhang et al. [20] calibrated the simulator from the perspective of absolute and relative validity. The simulator calibration was attained by setting up two experimental scenarios of free driving and following driving, and using mathematical statistics, regression methods, Wilcoxon test and non-parametric methods to compare the test point speed, driving distance, vehicle position, reaction time, risk perception and other parameters.

In terms of the current driving simulator validation methods, statistical testing is the main method, especially the Tukey and Anova tests. Speed, vehicle position, acceleration and deceleration are often considered as the validation parameters. By designing experimental scenes, conducting comparative experiments between vehicle and simulators is the main way to validate simulators. However, in the simulator validation experiment of road perception and driving interaction, the representativeness of complex road selection is quite critical. In addition, it is difficult to achieve the selection of complex roads and guarantee the safety of the experiment, as well as the efficiency and spread ability of the experiment being consequently low. According to the analysis of the above methods, considering the role of the road in the overall traffic, this paper selects the curve road as the criterion to evaluate the performance of the vehicle driving simulator, as the curve road condition can preferably evaluate the driving characteristics. In the comparison study of different curve 
radii, vehicle speed and trajectory are two main parameters that can comprehensively reflect driving characteristics [21,22]. On this account, this article validates the effectiveness of driving simulators based on speed from reliability and validity aspects, where reliability is an indicator of the consistency of test results, and validity accounts for the accuracy of test results [23]. In the curve test, the driver judges and manipulates the vehicle to pass the curve with different radii at certain speeds, and the speed value of each sampling point on the curve is equivalent to the test on the driver [24]. After that, the experiment's quality, i.e., the reliability of the experimental results, is evaluated. From this point of view, the reliability and validity theory can be used as a reference [25]. The driving trajectory is the specific performance of the driver's strategy and behavior of manipulating the vehicle. The similarity of the trajectory is an important reference index for investigating the steering behavior of the driver [26]. When analyzing the position parameters of the vehicle on the curve, the trajectory similarity model can be used to compare the simulator experiment. After the validation parameters and validation methods are determined, a method of using theoretical models instead of real vehicle experiments is proposed for easy acquisition of calibration data. In summary, this method can solve the problem that real complex scenes are difficult to realize in the simulator validation. Selecting the trajectory as the validation parameter can more comprehensively and intuitively reflect the simulator's curve driving state. Using speed model and trajectory model instead of real car experiment can improve the efficiency of simulator validation and lay a foundation for the standardization of simulator validation $[27,28]$.

Driving simulator technology has the advantage of being able to collaborate with multiple external vehicles at different speeds. It can also provide complex traffic environments and extreme test conditions. It further offers the advantage of safe and fast verification of motion strategies under complex operating conditions. Based on the above advantages, in recent years some car manufacturers and research institutes have started to experiment with driving simulators to promote research into automotive intelligence technologies. In particular, they have played an important role in the development and advancement of driverless car technology. For example, in January 2017, the US Department of Transportation designated 10 pilot units for testing unmanned vehicle technology, four of which were research institutes using driving simulators as a means of testing [29]. Therefore, as the advantages of driving simulators for applications in complex conditions increase, the need for driving simulator effectiveness under equivalent conditions is also increasing. The method proposed in this paper addresses the prediction of vehicle trajectories under extreme curves, small sample size and multi-dimensional metrics conditions. The proposed validation method addresses the reliability, robustness and generalisation of the simulator as a vehicle for the above research. The main contributions of this study adding to the literature are summarized as follows.

(1) A simulator validation method is designed under curved road conditions. By designing an experimental scenario for the simulator and conducting systematic analysis of the parameters and model measurement of the parameters, a comprehensive conclusion of simulator validation is attained. The method can effectively remedy the problem of insufficient research on the validation of simulators under complex road conditions.

(2) A simulator vehicle curve trajectory planning model is constructed, which leverages the Bi-LSTM neural network and employs the characteristic parameters of the driving behavior on the curves as input and the lateral offset of the vehicle trajectory as output. The model can replace the desired trajectory of a vehicle driving under complex road conditions, thereby reducing the cost of real vehicle parameter acquisition and improving the efficiency of simulator validation.

The remainder of this paper is structured as follows. Section 2 details the methods, and introduces the whole design framework. Section 3 explains the experimental data, and Section 4 discusses improvement of simulator curve track planning model, followed by the conclusion drawn in Section 5. 


\section{Methods}

\subsection{The Whole Design Framework}

By designing curves with different radii as experimental scenes, selecting the speed and trajectory as validation indicators, and exploiting the Kronbach's $\alpha$ reliability, split-half reliability, $r$ reliability, criterion-related validity and Cohen's $d$ exponent as the evaluation criteria [18], this paper completes the validation of the speed indicator of the driving simulator, and leverages the cosine similarity method to complete the validation of the driving simulator's trajectory. After converting the trajectory data into state variables, a body of sequence data of the driver's trajectory characteristics can be obtained. Based on the analysis of experimental data, the establishment of a curve trajectory planning model, i.e., a multi-bidirectional long-short term memory (Mul-Bi-LSTM) recurrent neural network (RNN), driven by human-like operation data is proposed [30,31]. Bi-LSTM is currently widely used in the prediction of sequence data, such as pedestrian trajectory prediction, intrusion detection, traffic speed prediction and traffic flow prediction [32-34]. Since the vehicle trajectory is sequence data and features a strong regularity, the Bi-LSTM neural network data-driven method enables to establish a vehicle trajectory planning model with high performance. The model results manifest that the proposed validation model can better replace the expected trajectory of a vehicle driving under complex road conditions and reduce the cost of collection of actual vehicle parameters. The whole design framework is shown in Figure 1. As can be found, the whole validation is conducted from the perspectives of speed and trajectory, which are respectively evaluated from reliability, validity, similarity and trajectory fit. In addition, a data-driven trajectory planning model is constructed to facility trajectory design.

\subsection{Experimental Design}

In this paper, the vehicle data are obtained through experiment under different horizontal curves, including longitudinal vehicle speed, longitudinal displacement, lateral displacement, longitudinal acceleration, vehicle position, accelerator pedal and brake pedal. The analysis of speed under curves, driving trajectory, theoretical vehicle speed and expected trajectory approximation are intensively analyzed to study the effectiveness of driving simulator based on vehicle-road interaction. As Table A1, a total of 27 drivers with different genders, ages, driving years and driving mileages are selected as the test subjects in the experiment, and they are numbered 1-27. Among them, all drivers are with corrected visual acuity of 1.0 and can skillfully complete the driving tasks. The experiment recruited 16 skilled drivers, which were test numbers $1,2,3,4,5,7,10,11,13,15,16,17,18,19,21$, and 26, including 11 male drivers and 5 female drivers. There are 11 new drivers, and the test numbers are $6,8,9,12,14,20,22,23,24,25$, and 27, including 7 male drivers and 4 female drivers. Drivers' age, driving experience, annual vehicle kilometers traveled total mileage, number and gender all meet the basic conditions of driving simulation experiments [35-37]. The experimental section is set as a two-way highway with no central separation zone and a length of nineteen kilometers. The design speed is $40 \mathrm{~km} / \mathrm{h}$ and the lane width is $3.5 \mathrm{~m}$. The experimental road types are composed of straight road sections and different horizontal curved road sections. The straight-line section is set between each characteristic road section (different horizontal curved sections), with a length of $700 \mathrm{~m}$, so that the driver can adjust their speed to enter the next curve with the expectation speed as the entry speed. The curve consists of a symmetrical basic type and simple type, mainly involving straight lines, circular curves and transition curves. 


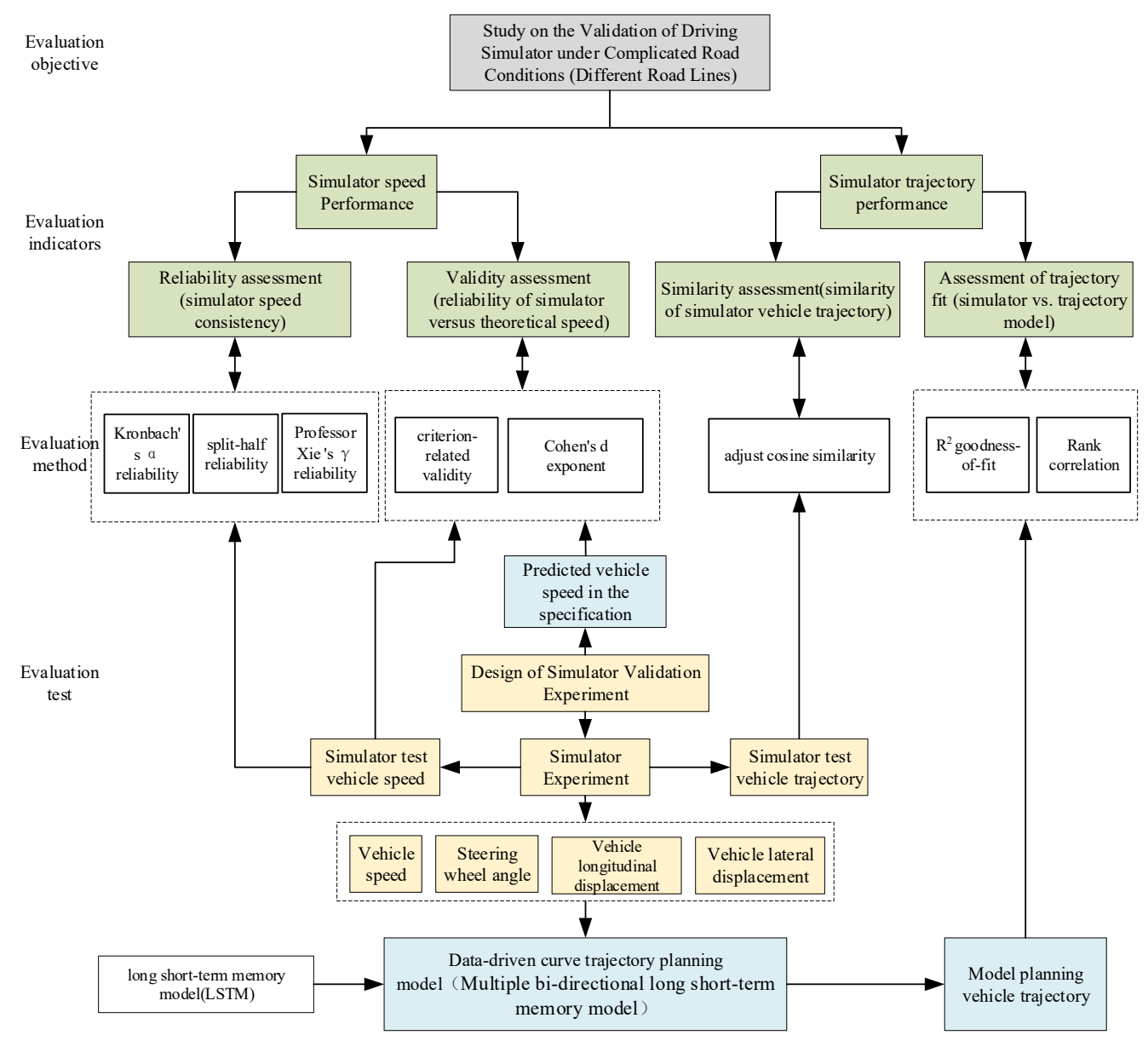

Figure 1. The whole design framework.

The circular curve setting considers the driving characteristics and the design speed to specify the minimum curve radius. According to Design Specification for Highway Alignment [38], the design radii include $55 \mathrm{~m}, 150 \mathrm{~m}, 250 \mathrm{~m}, 350 \mathrm{~m}, 450 \mathrm{~m}$ and $550 \mathrm{~m}$ and the transition curve is set to $35 \mathrm{~m}, 85 \mathrm{~m}, 135 \mathrm{~m}, 185 \mathrm{~m}, 235 \mathrm{~m}$ and $285 \mathrm{~m}$, considering centrifugal acceleration, driver operation response and reaction time, as well as visual conditions. Finally, a road design software is employed to devise the curve shape of the experimental characteristic curve. The virtual road environment scene is built based on our self-developed software [39]. The driving simulator system consists of two parts: the cockpit and the console. The cockpit portion was composed of an actual Jetta automobile equipped with all the necessary sensors and a monitoring system. Three cameras and one dual voice intercom system were installed in the cockpit, and the facial expressions, movements of the hands and feet, and sounds were transmitted to the recording system and the monitor to be stored for further study. Figure 2 shows the KMRTDS driving simulator and the test while driving. The visual scenario was presented on a large screen, providing an approximately $150^{\circ}$ horizontal view [40].
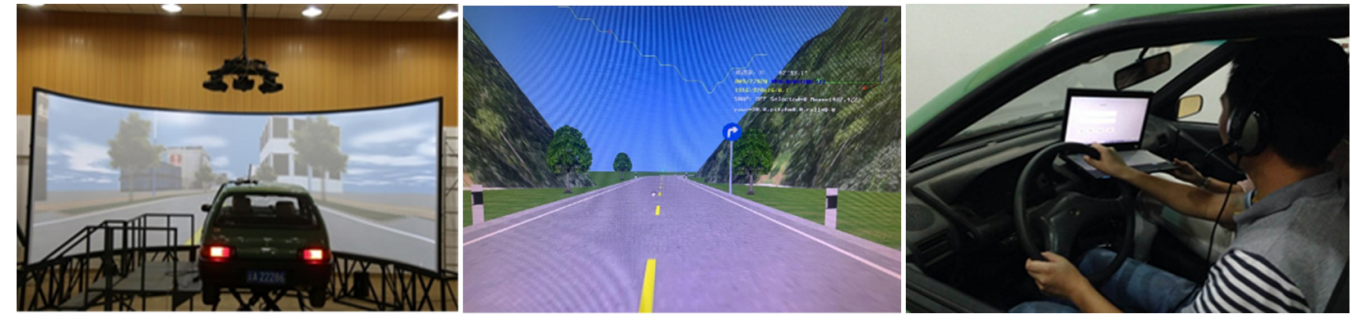

Figure 2. The KMRTDS driving simulator and the test while driving. 


\subsection{Experimental Data Preprocessing}

The driving simulator test can collect corresponding experimental data including simulation time, vehicle longitudinal acceleration, longitudinal velocity, lateral velocity, lateral acceleration, displacement, yaw rate, vehicle mileage, steering wheel angle, accelerator, pedal, brake pedal and gear steering. In order to facilitate the analysis of the driver's driving behavior in a curved road, it is necessary to preprocess the collected raw driving data. Since the start and end points of each trajectory data are slightly different, and the difference in vehicle speed leads to misalignment between the different original trajectory data. In the data processing, the form pile number is used as the curve landmark, and the curve landmark is set in each curve. A series of points is marked on the lane dividing line of the experimental road, and thus the distance between adjacent virtual landmarks is equal. The virtual landmarks are only used for calculations. Considering the length of the road and the number of sampling points at the same time, the interval of virtual landmarks in this paper is $1 \mathrm{~m}$. The sampling interval of the tested driving simulator is $50 \mathrm{~Hz}(20 \mathrm{~ms})$, and the sampling points will be different according to the speed of the vehicle. When analyzing the similarity of the vehicle trajectory, the experimental data should be distinguished according to the speed when entering the corner. As shown in Figure 3, for ease of calculation, a Cartesian coordinate system needs to be established by two mutually perpendicular coordinate axes, usually called the $x$-axis and $y$-axis, and each axis points to a specific direction. The middle $y$-axis is tangent to the lane dividing line, and the $x$-axis is perpendicular to the $y$-axis and points to the inner lane. The intersection of the two coordinate axes is the origin. The coordinate axes of these two different lines determine a plane, called $x y$-plane, i.e., the Cartesian plane. For easier understanding, this paper quotes the concept of lateral offset, which is the distance between the projection point of the vehicle center on the $x y$ plane and the form pile number. The specific calculation for the lateral offset can be formulated as:

$$
D_{P}=\sqrt{\left(X_{C A R}-X_{Z}\right)^{2}+\left(Y_{C A R}-Y_{Z}\right)^{2}},
$$

where $D_{P}$ is offset, $X_{C A R}$ is Longitudinal displacement of the vehicle, $X_{Z}$ is longitudinal displacement of pile number, $Y_{C A R}$ is vehicle lateral displacement, and $Y_{Z}$ is longitudinal displacement of pile number.

Driving direction: $\Uparrow$

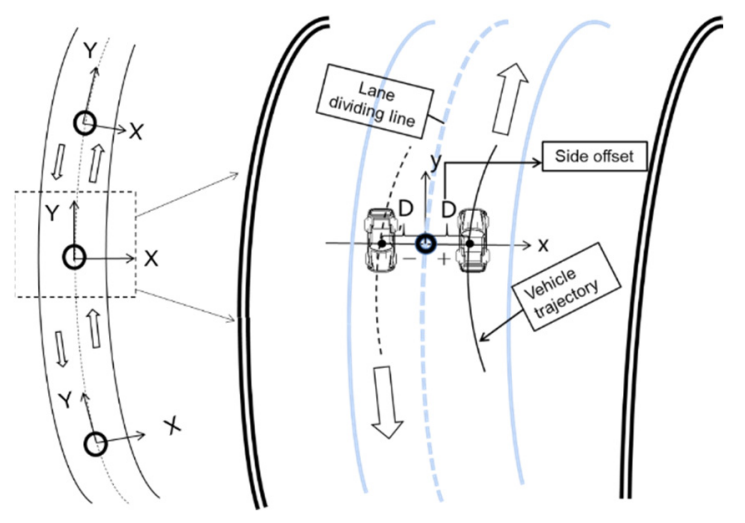

Figure 3. Driving simulation track diagram.

\section{Experimental Data Analysis}

\subsection{Speed Reliability Evaluation of Drivers in Curved Road Tests}

This paper randomly selects experimental curves 6 and 9 for specific analysis [37-39]. For curve $6(R=150 \mathrm{~m})$, according to the speed value of the vehicle entering the curve, the drivers are classified at $40 \mathrm{~km} / \mathrm{h}, 50 \mathrm{~km} / \mathrm{h}$ and $60 \mathrm{~km} / \mathrm{h}$, respectively, and there are seven testers who turn the curve at a speed of $60 \mathrm{~km} / \mathrm{h}$, and the driver numbers are 5,7 , 
$11,13,19,20$ and 23 . The testers who pass the curve road with $50 \mathrm{~km} / \mathrm{h}$ are No. 4, 8, 9, 10, 15, 16, 17, 18, 21, 26 and 27. There are 8 testers, i.e., No. 1, 2, 6, 12, 14, 22, 24 and 25, who cross the corner with $40 \mathrm{~km} / \mathrm{h}$. According to the speed analysis, it can be found that the drivers who turn at $60 \mathrm{~km} / \mathrm{h}$ are generally skilled drivers. Only tester 23 is classified as a new driver but with 4 years of driving experience of 50,000 kms. In this research, this can be classified as skilled driver. In the $50 \mathrm{~km} / \mathrm{h}$ cornering vehicle, only testers 8,9 and 27 are novice drivers, and in the $40 \mathrm{~km} / \mathrm{h}$ cornering vehicle, drivers $6,12,14,22,24$ and 25 are identified as novices. This further manifests as better performance of the skilled drivers when cornering. The related results are shown in Figure 4 . As can be observed, the vehicle entering a curve with the speed of $50 \mathrm{~km} / \mathrm{h}$ and $60 \mathrm{~km} / \mathrm{h}$ exhibits better speed change law, i.e., first deceleration and the consequent acceleration, and the vehicle entering the curve with the speed of $40 \mathrm{~km} / \mathrm{h}$ basically presents the characteristic of gradual acceleration after entering the curve. This can be explained by the reason that the initial speed of $50 \mathrm{~km} / \mathrm{h}$ and $60 \mathrm{~km} / \mathrm{h}$ is mainly adopted by skilled drivers, and it can also be proved that skilled drivers possess better turning maneuverability. The novice drivers mainly take the speed of $40 \mathrm{~km} / \mathrm{h}$ to cross the curve. Through analyzing the forward movement data, it can be found that the novice drivers tend to slow down before the curve due to tight cornering, therefore, the vehicle speed is reduced to the designed speed in the turning phase. Overall, the skilled drivers show a higher speed when entering curve 6 . By contrast, when the novice drivers enter the curve, the speed trend basically maintains the characteristics of deceleration first and consequent acceleration.

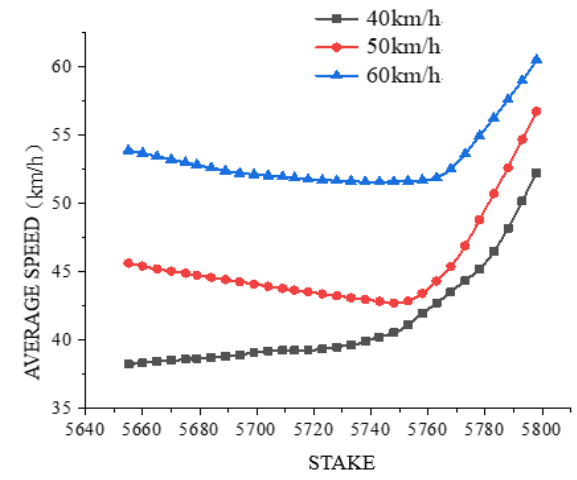

(a)

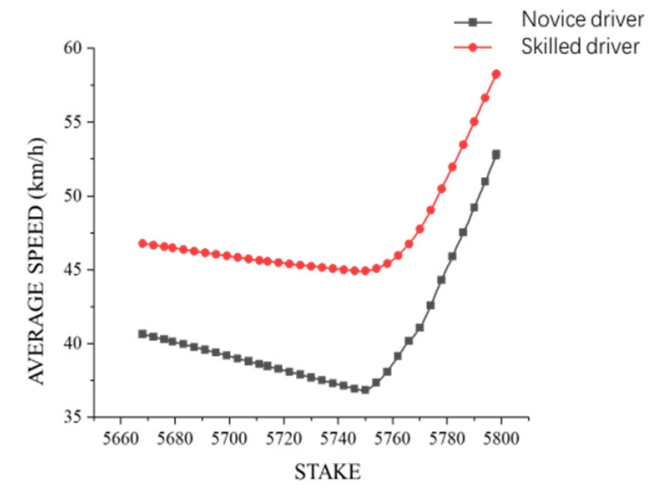

(b)

Figure 4. Speed analysis under curve 6. (a) Average velocity distribution; (b) Novice and skilled velocity distribution.

For curve $9(\mathrm{R}=55 \mathrm{~m})$, the drivers are classified according to the critical turning speeds of $30 \mathrm{~km} / \mathrm{h}, 40 \mathrm{~km} / \mathrm{h}$ and $50 \mathrm{~km} / \mathrm{h}$. Tester 11 , who turns with the speed of $60 \mathrm{~km} / \mathrm{h}$, can be excluded due to higher risk driving; and there are 8 testers, including testers $7,13,16$, 20, 22, 24, 25 and 26, turning with the speed of $50 \mathrm{~km} / \mathrm{h}$. Among them, the testers 20, 22, 24 and 25 are novice drivers. There exist 13 testers, including testers 1, 4, 5, 6, 8, 12, 14, 15, $17,18,19,21$ and 23, turning with the speed of $40 \mathrm{~km} / \mathrm{h}$, and among them the testers 6,8 , 12,14 , and 23 are novice drivers. Testers $3,9,10$ and 27 turn with the speed of $30 \mathrm{~km} / \mathrm{h}$, and tester 2 turn with the speed of $20 \mathrm{~km} / \mathrm{h}$. Since there is only one case for the tester with high speed of $60 \mathrm{~km} / \mathrm{h}$ and low speed of $20 \mathrm{~km} / \mathrm{h}$, it is not discussed and instead considered as abnormal data. As shown in Figure 5, the vehicles entering a curve at the speed of $40 \mathrm{~km} / \mathrm{h}$ and $50 \mathrm{~km} / \mathrm{h}$ exhibit a better speed change law, i.e., first deceleration and following acceleration. The vehicles entering a turn at the speed of $30 \mathrm{~km} / \mathrm{h}$ basically present gradual acceleration after entering the curve. According to the analysis of testers who use different speeds to enter a curve, there is no specific distribution law between skilled drivers and novice drivers. Through the analysis of the forward movement data, the drivers who enter the curve with the speed of $30 \mathrm{~km} / \mathrm{h}$ tend to slow down before the curve, and the speed drops to the minimum value at the turning phase. On the whole, the turning 
speed of skilled drivers looks basically the same that of the novice drivers in curve 9, and the skilled drivers generally maintain the operations of first deceleration and the following acceleration, while the novice drivers raise more significant deceleration trend.

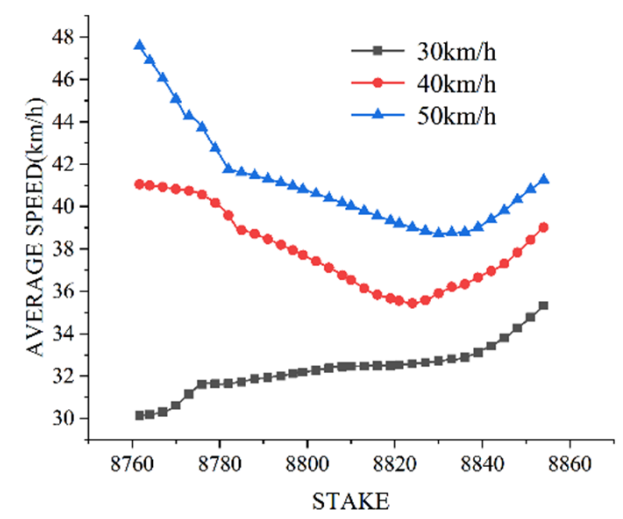

(a)

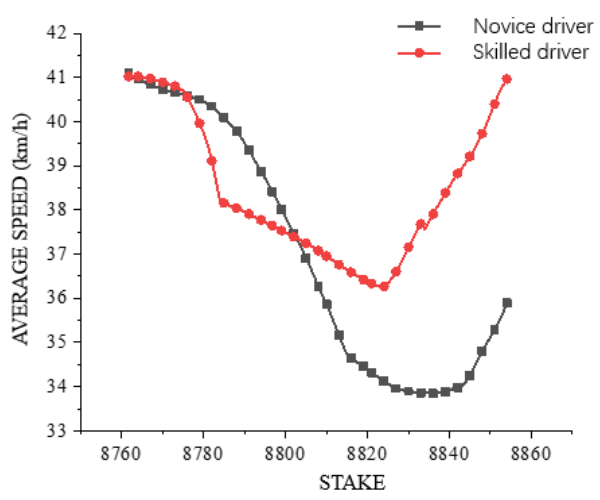

(b)

Figure 5. Trajectory analysis of curve 9: (a) average velocity distribution; (b) novice and skilled velociy distribution.

Based on the analysis of the vehicle speed, the reliability coefficients of curves 6 and 9 are further obtained. According to the validation method of the curve reliability, the Kronbach's $\alpha$ reliability, split-half reliability and $r$ reliability were selected to evaluate the simulator speed [25], as shown in the following equations. The Kronbach's $\alpha$ reliability can be presented as:

$$
\alpha=\frac{k}{k-1}\left[\frac{S_{X}^{2}-\sum S_{X_{i}}^{2}}{S_{X}^{2}}\right]=\frac{k}{k-1}\left[1-\frac{\sum S_{X_{i}}^{2}}{S_{X}^{2}}\right]
$$

where $k$ is the number of form stakes, $S_{X}^{2}$ is the total variance of the sampled vehicle speed, and $S_{X_{i}}^{2}$ is the speed variance of the driver at the $X_{i}$ form stake. The split-half reliability can be expressed by Spearman-Brown coefficient, as:

$$
r_{x x}=\frac{2 \times \frac{\sum X_{1} X_{2} / n-\overline{X_{1} X_{2}}}{S_{x_{1}} S_{x_{2}}}}{1+\frac{\sum X_{1} X_{2} / n-\overline{X_{1} X_{2}}}{S_{x_{1}} S_{x_{2}}}}=\frac{2\left(\sum X_{1} X_{2} / n-\overline{X_{1} X_{2}}\right)}{S_{x_{1}} S_{x_{2}}+\left(\sum X_{1} X_{2} / n-\overline{X_{1} X_{2}}\right)}
$$

where $r_{x x}$ is the reliability coefficient of the simulator curve experiment, $X_{1}$ is the oddnumbered pile speed sum of the curve, $X_{2}$ is the even-numbered pile speed sum of the curve, $\overline{X_{1}}$ is the mean value of the odd-numbered pile speed sum of the curve, $\overline{X_{2}}$ is the mean value of the sum of even-numbered station speeds in the curve, $n$ is the number of test drivers, $S_{X_{1}}$ is the standard deviation of the odd-numbered station speed sum and $S_{X_{2}}$ is the standard deviation of the even-numbered station speed sum. The $r$ reliability [41] can be formulated, as:

$$
r=1-\frac{\sqrt{k \sum_{i=1}^{k} S_{i}{ }^{2}-S^{2}} \times 3.92}{\sqrt{k-1}\left(X_{\max }-X_{\min }\right)}
$$

where $S^{2}$ is the total variance of the sum of speeds in the curve, $S_{i}{ }^{2}$ is the variance of the speed under the $i$ form of stake, $k$ is the number of form of stakes, $X_{\max }$ and $X_{\min }$ is the highest and lowest values of the sum of speeds. In terms of these coefficients, the result is shown in Table 1. By selecting the experimental data of curves with different radii and obtaining the reliability coefficient values according to the reliability model, it is found that 3 types of reliability coefficients for curve 6 meet the experimental reliability requirements; whereas for the curve 9, the $\alpha$ reliability coefficient and Spearman-Brown coefficient meet the requirements, but the $r$ reliability coefficient is low. Note that when 
evaluating the reliability of the test, the $r$ coefficient should be generally higher than 0.70 , the other reliability coefficients should be generally higher than 0.80 [41-43]. Therefore, the data consistency of curve 9 is biased simply from the perspective of $r$ reliability coefficient. Meanwhile, the reliability coefficients of the data are improved after data cleaning. Therefore, the difference between testers is a key factor that should be taken into consideration. Furthermore, through the comparison of reliability coefficients, the reliability of the tested driving simulator in small-radius turns is low, due to the insufficient follow-up of the steering scene when testers turn in the simulator. In this context, the testers lack a full understanding of the lateral scene and cannot accurately complete the driving operation.

Table 1. Reliability coefficient results of curve 6 and cure 9 .

\begin{tabular}{ccccc}
\hline Reliability & $\begin{array}{c}\text { Curve 6 } \\
\text { (Outliers } \\
\text { Included) } \\
(\boldsymbol{R}=\mathbf{1 5 0} \mathbf{~})\end{array}$ & $\begin{array}{c}\text { Curve 6 } \\
\text { (Outliers } \\
\text { Excluded) } \\
(\boldsymbol{R}=\mathbf{1 5 0} \mathbf{m})\end{array}$ & $\begin{array}{c}\text { Curve 9 } \\
\text { (Outliers } \\
\text { Included) } \\
(\boldsymbol{R}=\mathbf{5 5} \mathbf{~})\end{array}$ & $\begin{array}{c}\text { Curve 9 } \\
\text { (Outliers } \\
\text { Excluded) } \\
(\boldsymbol{R}=\mathbf{5 5} \mathbf{~ m )}\end{array}$ \\
$\begin{array}{c}\alpha \text { reliability } \\
\text { Spearman-Brown }\end{array}$ & 0.908 & 0.932 & 0.807 & 0.807 \\
coefficient & 0.912 & 0.982 & 0.885 & 0.894 \\
$\begin{array}{r}r \text { reliability } \\
\text { Mean reliability }\end{array}$ & 0.714 & 0.844 & 0.59 & 0.61 \\
\hline
\end{tabular}

\subsection{Verification of Driving Speed in Curve Test}

Based on the basic information of the experimental road, the operating speed is theoretically calculated according to the speed prediction model detailed in the specification [44], as:

$$
\begin{array}{r}
v_{\text {middle }}=-244.123+0.6 v_{\text {in }}+40 \ln \left(R_{\text {now }}+500\right), v_{\text {in }} \in[30,120], R_{\text {now }} \in[55,600] \\
v_{\text {out }}=-183.092+0.7 v_{\text {middle }}+30 \ln \left(R_{\text {front }}+500\right), v_{\text {middle }} \in[30,120], R_{\text {front }} \in[55,600]
\end{array}
$$

where $v_{\text {in }}$ is the running speed at the entrance of the curve, $v_{\text {middle }}$ is the running speed at the midpoint of the curve, $v_{\text {out }}$ is the running speed at the exit of the curve, $R_{\text {now }}$ is the radius of current curve and $R_{\text {front }}$ is the radius of the curve to be driven into when the front is a straight line, $R_{\text {front }}=600 \mathrm{~m}$. If $R_{\text {front }}>5 R_{\text {now }}, R_{\text {front }}$ is set to $5 R_{\text {now }}$.

Through the calculation and comparison of the experimental data, the test values and predicted values of sampling points are shown in Figure 6. To intuitively analyze the data sampled from the starting point, midpoint and end point of the curve, the test data of the simulator is compared with the theoretical calculation value of the speed prediction model in the design specification, and it can be found that the overall change trend of speed is consistent, while there exists large speed deviation at the entrance of individual curves. The results of the simulator experiment are generally higher than the theoretical values. The points where the simulated value is lower than the theoretical speed appear in curves $9,10,14$, and 16 . The curve radius query results show that the radius of the curves 9 and 16 is $55 \mathrm{~m}$, and the radius of the curve 14 is $150 \mathrm{~m}$, demonstrating that the driver in large-radius curves is more aggressive in the driving simulator; however, the driver tends to be more cautious in the small-radius sharp curves, which is related to the lack of effectiveness provided by the simulator to drivers in sharp turns, thus complying with the conclusion from the reliability evaluation that the driver test performs with low reliability in small-radius curves. 


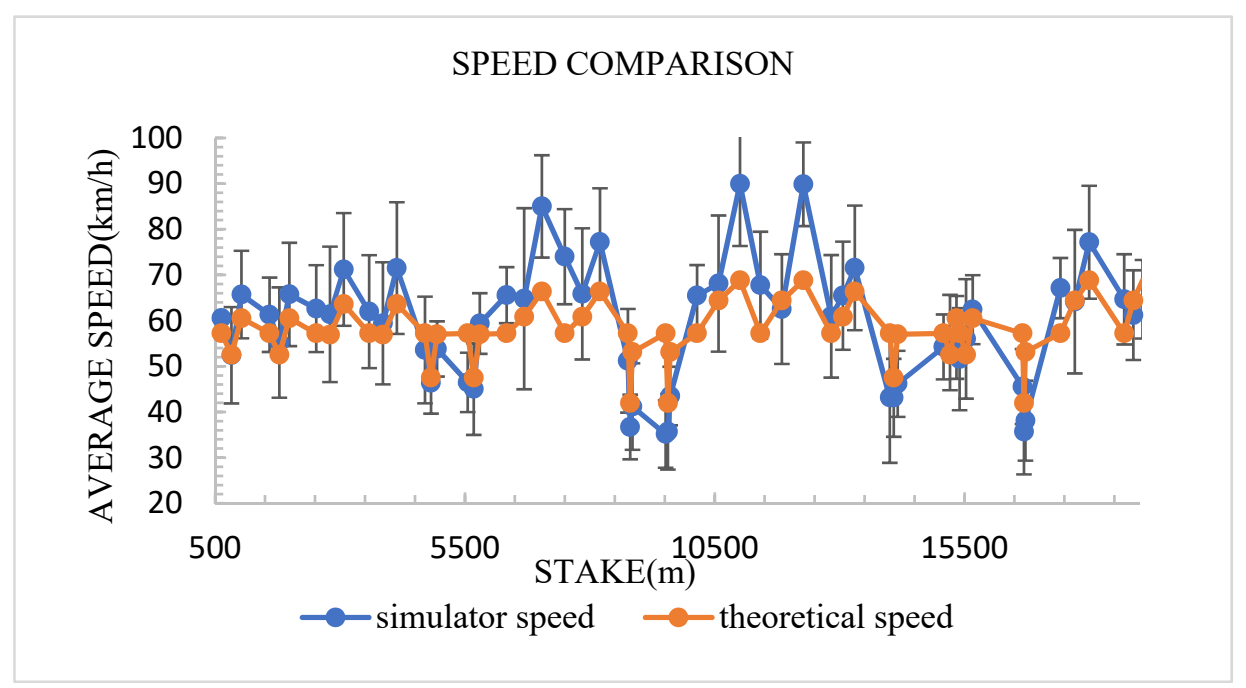

Figure 6. Speed comparisons of curve sampling point.

From a quantitative point of view, according to the criterion-related validity theory, the Pearson correlation method is employed to obtain the validity coefficient of 0.802 between the simulator data and the theoretical data. Based on the obtained correlation coefficient, the Student's $t$ testis performed. The Student's $t$ test is to use the $t$ distribution theory to infer the probability of the difference, so as to compare whether the difference between two averages is significant. According to the result of the paired sample test, when the preset significance level $\alpha$ is $0.05, t$ is 0.781 , and the significance level is 0.438 . Hence, it can be considered that the difference between the two groups of data is not significant. The comprehensive validity and statistical test prove that the driving simulator is effective in simulating turning road conditions. Based on the validity analysis, according to the meta-analysis method, the component difference $t$ is adopted to calculate Cohen's $d$ index as:

$$
d=\frac{2 t}{\sqrt{d f}}
$$

By substituting the test value into (7), we can get $d=0.2$. According to the Cohen's effect level classification, the effect level is low at this time, this is mainly due to the large difference in the test value of the small-radius curve based on the subjective analysis of the test value. By excluding the small-radius curve data for verification, we can get $t=3.659$, $d f=47$ and $d=1.07$, highlighting that the simulator test owns large effect level. Therefore, it can be explained that the simulator generates a large effect in turning conditions with a radius of greater than $150 \mathrm{~m}$, but with low effect for small-radius curves. The test simulator has a high validity coefficient under turning road conditions when the radius of the curve is greater than $150 \mathrm{~m}$. However, the validity on small radius curves with radius less than $55 \mathrm{~m}$ is low and needs to be improved.

\subsection{Verification of Vehicle Trajectory in Curve Test}

According to the designed experimental scheme, the trajectory data of the vehicle at the selected curve $6(R=150 \mathrm{~m})$ and curve $9(R=55 \mathrm{~m})$ is obtained using the typical vehicle trajectory planning RRT algorithm, and under the same virtual landmark, the lateral offset of the path planned by RRT algorithm at the curve is gained after transformation. The RRT algorithm is a growing tree search algorithm based on random sampling, which is widely used in robot path planning [45]. The experimental vehicle trajectory and RRT predicted trajectory for curves 6 and 9 are illustrated in Figures 7 and 8, and the trajectory similarity values are shown in Tables $2-4$. 


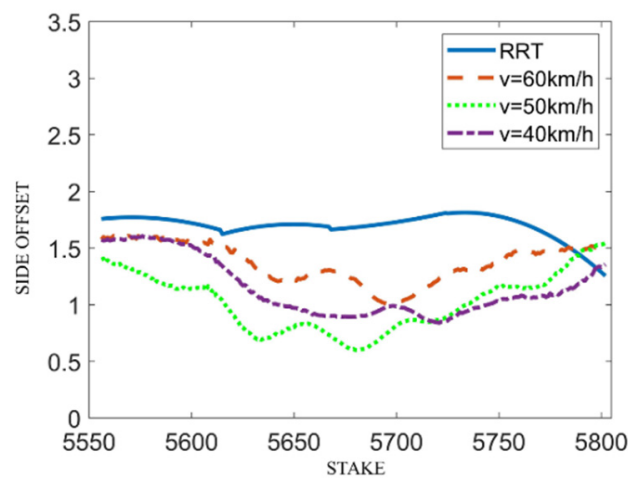

Figure 7. Curves of RRT lateral offset at different speeds in Turn 6.

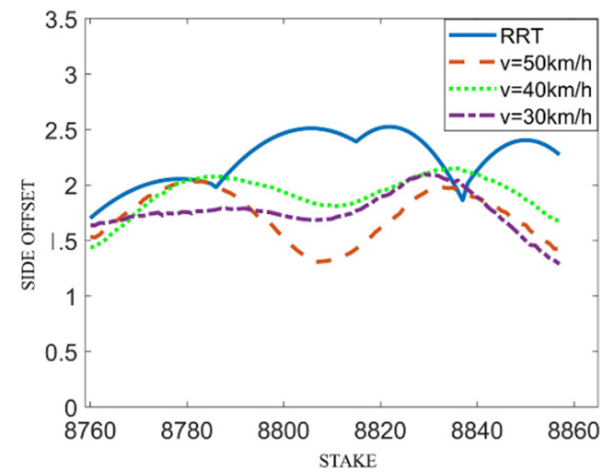

Figure 8. Curves of RRT lateral offset at different speeds in Turn 9.

Table 2. Trajectory similarity of different types of drivers.

\begin{tabular}{cccc}
\hline & Cure $6(60 \mathrm{~km} / \mathbf{h})$ & Cure $6(40 \mathrm{~km} / \mathbf{h})$ & Cure $\mathbf{9}(\mathbf{4 0} \mathbf{~ k m} / \mathbf{h})$ \\
\hline Skilled driver & 0.9715 & - & 0.9956 \\
Novice driver & - & 0.8583 & 0.9785 \\
\hline
\end{tabular}

$-:$ No comparison possible.

Table 3. Cosine similarity of different vehicle speeds and RRT trajectory adjustment in turn 6.

\begin{tabular}{ccccc}
\hline & RRT & $\mathbf{6 0}$ & $\mathbf{5 0}$ & $\mathbf{4 0}$ \\
\hline RRT & - & -0.2156 & -0.3718 & -0.0791 \\
60 & - & - & 0.7909 & 0.8252 \\
50 & - & - & - & 0.6732 \\
\hline
\end{tabular}

Table 4. Cosine similarity of different vehicle speeds and RRT trajectory adjustment in turn 9.

\begin{tabular}{ccccc}
\hline & RRT & $\mathbf{5 0}$ & $\mathbf{4 0}$ & $\mathbf{3 0}$ \\
\hline RRT & - & -0.539 & 0.2187 & 0.0041 \\
50 & - & - & 0.6143 & 0.5072 \\
40 & - & - & - & 0.6475 \\
\hline
\end{tabular}

For curve 6, the vehicle with higher speed leads to larger fluctuation of the lateral offset and tends to be driven closely to the center line of the lane at the midpoint of the curve. Thus, the trend of skilled drivers driving in the middle of the road is more obvious. According to the classification of drivers with different speeds in curve 6 , all those who turn at the speed of $60 \mathrm{~km} / \mathrm{h}$ are skilled drivers, and those who turn at the speed of $40 \mathrm{~km} / \mathrm{h}$ are basically novice drivers and a few cautious skilled drivers. The skilled drivers feature more advanced driving skills and can judge turns more accurately and turn at the speed of $60 \mathrm{~km} / \mathrm{h}$. The trajectory similarity between the skilled drivers is as high as 0.9715 . The novice drivers perform unskilled and cautious driving behavior and basically control the 
speed at a low level of $40 \mathrm{~km} / \mathrm{h}$ to pass through the turn. The trajectory similarity between novice drivers is lower than that of skilled drivers, while the overall similarity also reaches 0.8583. In the driving simulator, the trajectory similarity of vehicles with the speed of $40 \mathrm{~km} / \mathrm{h}$ and $60 \mathrm{~km} / \mathrm{h}$ is the largest, reaching 0.8252 , proving that novice drivers with low speed can reach a trajectory similarity to that of skilled drivers with the speed of $60 \mathrm{~km} / \mathrm{h}$. In curve 9, the trajectory similarity between the novice and the skilled drivers who turn at the same speed of $40 \mathrm{~km} / \mathrm{h}$ reach 0.9785 and 0.9956 , respectively, and the trajectory similarity of the skilled drivers is higher than that of the novice drivers. The vehicles with the speed of $30 \mathrm{~km} / \mathrm{h}$ and $40 \mathrm{~km} / \mathrm{h}$ own the highest trajectory similarity of 0.6475 , but from the analysis of the trajectory cosine similarity, the overall trajectory of the driving simulator in curve 9 fluctuates prominently; meanwhile, the trajectory similarity of vehicles with different speeds is markedly low. Through comprehensive analysis of the RRT trajectory calculation process under the designed curve radii, the comparison between the experimental vehicle trajectory and RRT trajectory, and between the similarity values, it can be concluded that the path point planned by RRT is quite close to the obstacle, which is the edge of the road. When planning the curve path, there emerges a phenomenon of excessive turning. The trajectory predicted by RRT in curve 6 and the trajectory predicted by the driving simulator are both negative, manifesting that the trajectories of the two are opposite. The trajectory predicted by RRT in curve 9 and the trajectory predicted by the driving simulator with the speed of $50 \mathrm{~km} / \mathrm{h}$ are negative, which proves that the trajectories of the two vehicles are opposite at this speed, while the vehicle trajectories with other speeds are consistent, but the similarity is not high.

\section{Improvement of Simulator Curve Track Planning Model}

Through the analysis of the experimental data, it is concluded that RRT does not consider the actual driving speed, steering and other vehicle behavior factors in the curve trajectory planning process. It is necessary to establish a curve trajectory planning model driven by human-simulating operation data to provide a trajectory calibration reference for the simulator's validaton under turning conditions.

\subsection{Data-Driven Modeling Method}

\subsubsection{Long Short-Term Memory Recurrent Neural Network}

Since the lateral offset during vehicle driving is time series data, a supervised learning model can be constructed to predict this variable. The model needs to use time series data including the longitudinal displacement of the vehicle, the lateral displacement of the vehicle, the longitudinal speed of the vehicle and the steering wheel angle. The time series data itself is not only affected by the previous input characteristics, but also by the input characteristics at previous moment. In view of the above characteristics, this paper adopts a neural network with time series properties for data fitting and model construction. As shown in Figure 9, when the recurrent neural network (RNN) processes sequence data, it searches the data before the current state, saves it in the model and exploits it in the current network output, making it suitable for predicting the lateral offset of vehicles with time series. In actual calculations, in order to reduce the complexity of the algorithm and increase the inference speed of the model, it can be assumed that the current state is related to the previous states. The RNN will always receive the input $x_{t}$ and loop inside the model. Each calculation will use the previous calculation information.

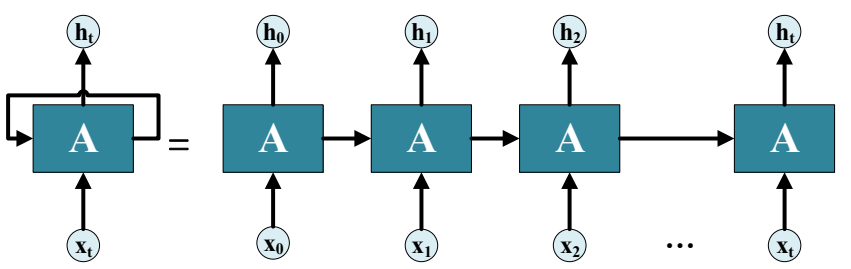

Figure 9. Schematic diagram of RNN. 
From the perspective of the data set, the sampling frequency of vehicle operation is relatively high, and the current input of the model has a relationship with the input within a long period of time. When the RNN model processes long-period data, as the input pre-time sequence state is too much, the gradient will explode and disappear due to long-term dependence. Therefore, long short-term memory (LSTM) RNN is adopted to enhance the model's ability to learn long-term dependence [46]. Figure 10 presents the network structure of LSTM, where the cell state $C_{t}$ represents the memory information of the model at time $t$. The forgetting gate $f_{t}$ takes the previous sequence $h_{t-1}$ and the current sequence $x_{t}$ as input, and obtains the forgotten and retained part of the data information through the activation function. The sigmoid function is employed as activation function, which is close to 0 or 1 within the range of data values. The forgetting gate is described as:

$$
f_{t}=\sigma\left(W_{f} \cdot\left[h_{t-1}, x_{t}\right]+b_{f}\right)
$$

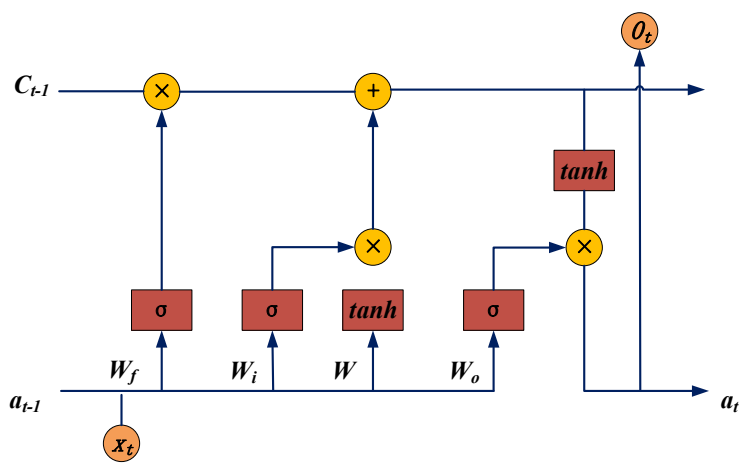

Figure 10. LSTM network structure diagram.

The input gate needs to process the input information of the current sequence, and determine the data to update the model, so as to update the model state. The sigmoid function is used to determine the information characteristics of the data which are added to the model, and the new characteristic information is converted into the added data information using the tanh function, as:

$$
\begin{gathered}
i_{t}=\sigma\left(W_{i} \cdot\left[h_{t-1}, x_{t}\right]+b_{i}\right) \\
\bar{C}_{t}=\tanh \left(W_{c} \cdot\left[h_{t-1}, x_{t}\right]+b_{c}\right)
\end{gathered}
$$

The update gate updates the cell status with the forget gate and output gate information received. The update gate $f_{t} * C_{t-1}$ represents the information removed from the model cell, and $i_{t} * C_{t-1}$ represents the new data information of the model cell. The update gate is formulated, as:

$$
C_{t}=f_{t} * C_{t-1}+i_{t} * \bar{C}_{t}
$$

The output gate applies the sigmoid function to confirm the output content, and the tanh function to process the model cell content, then the output information is obtained by multiplying the two parts, as:

$$
\begin{gathered}
O_{t}=\sigma\left(W_{o} \cdot\left[h_{t-1}, x_{t}\right]+b_{o}\right) \\
h_{t}=O_{t} * \tanh \left(C_{t}\right)
\end{gathered}
$$

where the sigmoid function is the logistic function, which is an S-shaped growth curve in biology. In practice, because of the monotonically increasing feature of the function, it is often adopted as the activation function of the neural network, which can map the input variables of the neural network to $[0,1]$, as:

$$
\sigma=\frac{1}{1+e^{-x}}
$$


The tanh function is one of the hyperbolic functions. The tanh function overcomes the central asymmetry of the sigmod function, and its value range is $[-1,1]$, as:

$$
\tanh (x)=\frac{e^{x}-e^{-x}}{e^{x}+e^{-x}}
$$

The forget gate, update gate and output gate all adopt non-linear activation functions to enhance the learning ability of the network and enable the model to master highdimensional complex data.

\subsubsection{Bi-LSTM}

The Bi-LSTM is an extension of traditional LSTM, which can improve the performance of the model to solve the time series regression problem. Based on the characteristics of the lateral offset, the prediction may need to be jointly determined by several previous inputs and several subsequent inputs, which will be more accurate. Therefore, the BiLSTM is employed in this study, which uses time series processing for the past and future bidirectional data, trains the front and back time series data added by the network model, and leverages forward and backward bidirectional LSTM to model the data. The forward LSTM model performs forward calculations on the data from 1 to $t$, saves the forward output of each sequence data time; and the backward LSTM model performs reverse calculations on the data from $t$ to 1 , and saves the backward output at each sequence data time. By synthesizing the forward and backward LSTM models to obtain the final output [47], we can yield:

$$
\begin{gathered}
h_{t}=f\left(w_{1} x_{i}+w_{2} h_{t-1}\right) \\
h_{t}^{\prime}=f\left(w_{3} x_{t}+w_{5} h_{t+1}^{\prime}\right) \\
O_{t}=g\left(w_{4} h_{t}+w_{6} h_{t}^{\prime}\right)
\end{gathered}
$$

\subsubsection{Multilayer Perceptron}

MLP, also a member of artificial neural networks, is based on bionics, which proposes the perception of information from the perspective of human organ perception, and the acquisition is built as a prototype. It was proposed by Frank Rosenblatt in 1958 [48].

\subsubsection{Prediction Results and Model Validation}

In order to evaluate the predictive performance of the model, seven error validation indicators are considered to establish a multi-indicator fusion evaluation plan [49], including:

(a) Goodness of fit $\left(R^{2}\right)$

$$
R^{2}=\frac{S S R}{S S T}=\frac{\sum_{i=1}^{n}\left(\hat{y}_{i}-\bar{y}\right)^{2}}{\sum_{i=1}^{n}\left(y_{i}-\bar{y}\right)^{2}}=1-\frac{S S E}{S S T}
$$

(b) Mean of absolute error (Error Mean)

$$
\mu=\frac{1}{n} \sum_{i=1}^{n}\left(\hat{y}_{i}-y_{i}\right)
$$

(c) Standard deviation of absolute error (Error Std)

$$
\sigma=\sqrt{\frac{1}{n} \sum_{i=1}^{n}\left(\hat{y}_{i}-y_{i}-\mu\right)^{2}}
$$

(d) Mean square error (MSE)

$$
M S E=\frac{1}{n} \sum_{i=1}^{n}\left(\hat{y}_{i}-y_{i}\right)^{2}
$$


(e) Root mean square error (RMSE)

$$
R M S E=\sqrt{\frac{1}{n} \sum_{i=1}^{n}\left(\hat{y}_{i}-y_{i}\right)^{2}}
$$

(f) Normalized root mean square error (NRMSE)

$$
\text { NRMSE }=\frac{\sqrt{\frac{1}{n} \sum_{i=1}^{n}\left(\hat{y}_{i}-y_{i}\right)^{2}}}{\bar{y}}
$$

(g) Rank Correlation

$$
r_{s}=1-\frac{6 \sum d_{i}^{2}}{n\left(n^{2}-1\right)}
$$

where $R^{2}$ is the goodness of fit, $y_{i}$ is the original trajectory data $(i=1,2, \cdots, n), \hat{y}_{i}$ is the predicted value, $\bar{y}$ is the average value, SSR is the regression sum of squares, SST is the sum of square deviations, and SSE is the residual sum of square. $r_{S}$ is the rank correlation coefficient, $d_{i}$ is the level difference of each pair of samples of the two variables, $x_{i}$ is the difference between the variable and $y_{i}$, and $n$ is the sample size.

\subsubsection{Model Training}

In order to reflect the performance advantages of Bi-LSTM model and avoid parameter overfitting, the structural performance of Bi-LSTM model is set manually to make it optimal. Twenty-four sets of crossover experiments in the form of number of hidden layers $\times$ number of traversal rounds $\times$ batch size were designed for testing as shown in Table 5 . The model contains four classes of hidden layer structures, with only one hidden layer in class 1 and a number of hidden layer cells of 100, denoted as $h_{100}$. Class 2 has two hidden layers, both with a number of neurons of 100, denoted as $h_{100} \times h_{100}$. The remaining two classes are denoted as $h_{100} \times h_{100} \times h_{75}$ and $h_{100} \times h_{100} \times h_{75} \times h_{75}$.

Table 5. The structure of the cross-experiment.

\begin{tabular}{cccc}
\hline Hidden Layers & Iteration Rounds & Lot Size & Model Number \\
\hline$h_{100}$ & & $1-6$ \\
$h_{100} \times h_{100}$ & 100-200, Step length & 32-128, & $7-12$ \\
$h_{100} \times h_{100} \times h_{75}$ & is 100 & Step length is 32 & $13-18$ \\
$h_{100} \times h_{100} \times h_{75} \times h_{75}$ & & & $19-24$ \\
\hline
\end{tabular}

The training process sets the base learning rate $\beta$ to 0.005 and the dropout value is 0.5 . Using the Adam iterative optimization algorithm [50], the learning rate of each parameter is dynamically adjusted using first-order moment estimation and second-order moment estimation of the gradient with the following equations.

$$
\begin{gathered}
m_{t}=\lambda \times m_{t-1}+(1-\lambda) \times g_{t} \\
n_{t}=\gamma \times n_{t-1}+(1-\gamma) \times g_{t}^{2} \\
\hat{m}_{t}=\frac{m_{t}}{1-\lambda^{t}} \\
\hat{n}_{t}=\frac{n_{t}}{1-\gamma^{t}} \\
\theta_{t}=\theta_{t-1}-\frac{\hat{m}_{t}}{\sqrt{\hat{n}_{t}}+\varepsilon} \times \beta
\end{gathered}
$$

where $g_{t}$ is the gradient of the time step, $m_{t}$ is the first-order moment estimate of $g_{t}$, which is the exponential moving average of $g_{t}, n_{t}$ is the second-order moment estimate of $g_{t}$, the 
exponential moving average of $g_{t}^{2}, \lambda$ and $\gamma$ is the exponential decay rate, $\hat{m}_{t}$ is the deviation correction for $m_{t}, \hat{n}_{t}$ is the deviation correction for $n_{t}, \theta_{t}$ is the parameter vector for time step $t, \beta$ is the learning rate, $\varepsilon$ is the residual term, the default is taken as $10^{-8}$.

After completing 24 sets of training tests, the distribution of $\bar{L}_{0.05}$ and $M_{R M S E}$ of the model is obtained. As the number of hidden layers increases, the $\bar{L}_{0.05}$ and $M_{R M S E}$ curves rise, and $\bar{L}_{0.05}$ and $M_{R M S E}$ of the group 4 experiments are at a lower level, i.e., the structural performance of the group 4 model is optimal. With the same number of hidden layers, $\bar{L}_{0.05}$ and $M_{R M S E}$ show a decreasing trend as the number of traversal rounds increases. Taking groups 1-6 as an example: the number of traversal rounds is 100 for groups 1-3 $\left(h_{100} \times 100 \times 32, h_{100} \times 100 \times 64, h_{100} \times 100 \times 128\right)$ and 200 for groups $4-6\left(h_{100} \times 200 \times 32\right.$, $\left.h_{100} \times 200 \times 64, h_{100} \times 200 \times 128\right)$. The change in the number of traversal rounds shows a significant decrease in the values of $\bar{L}_{0.05}$ and $M_{R M S E}$. In the case of the same hidden layer and the same number of traversal rounds, for example, with the increase of batch size, $\bar{L}_{0.05}, M_{R M S E}$ also tend to increase. Based on the above analysis, group 4 (with hidden layer of, lot size of 32 and number of traversal rounds of 200) is finally adopted as the optimal Bi-LSTM model.

\subsection{Model Verification and Testing}

In order to compare the prediction performance of LSTM, MLP, and Mul-Bi-LSTM, 28 sets of the same vehicle curve data are used to establish trajectory planning models, respectively. The input of Mul-Bi-LSTM model is the longitudinal displacement of the vehicle, the lateral displacement of the vehicle, the longitudinal speed of the vehicle, and the steering wheel angle and the output is the lateral offset. The input and output of LSTM and MLP models are all lateral offsets. After data processing, the simulation prediction result of single variable and Mul-Bi-LSTM input is obtained. Three sets of typical data are randomly selected for comparative analysis, as shown in Figures 11-13.

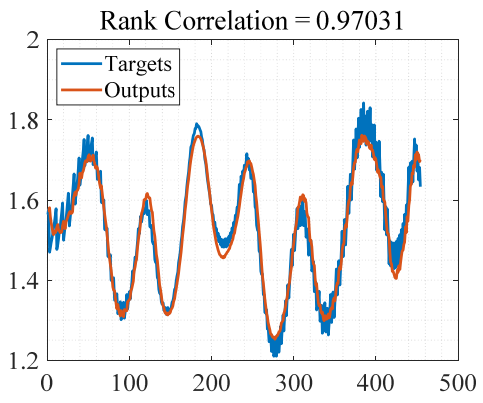

(a)

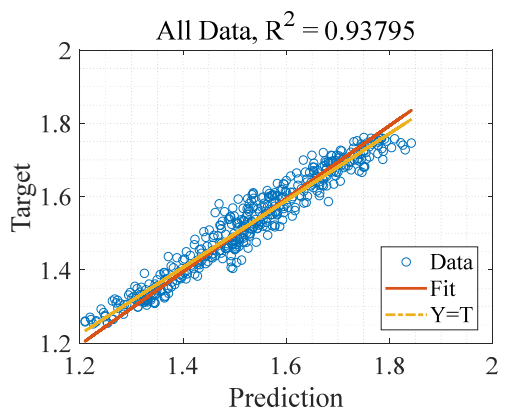

(d)

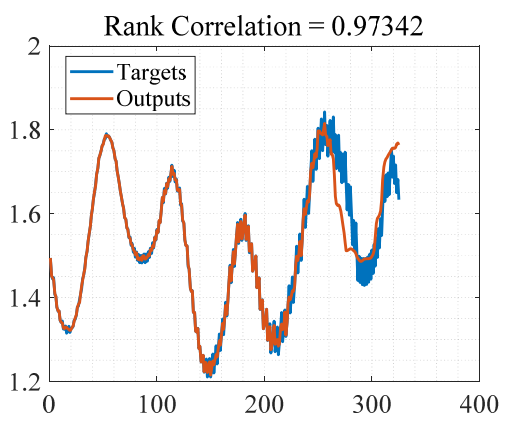

(b)

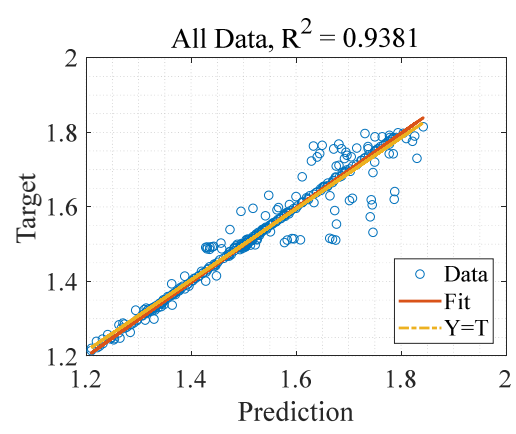

(e)

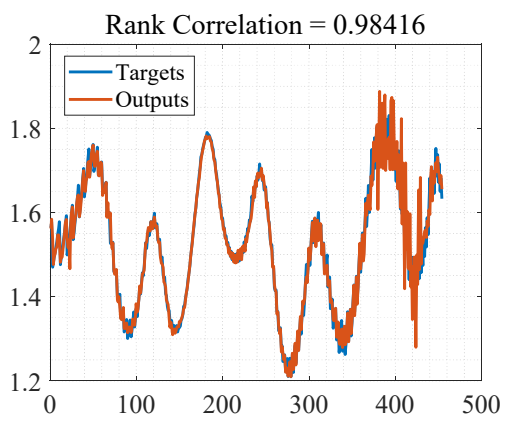

(c)

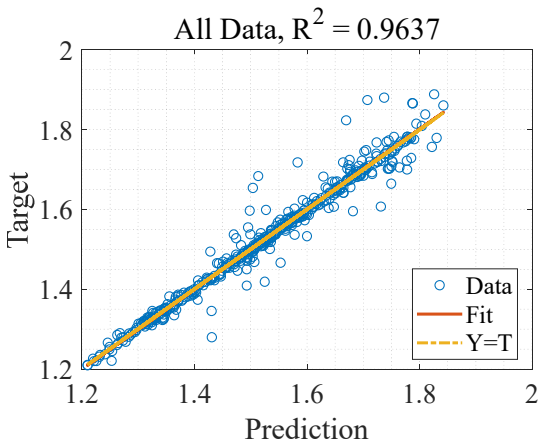

(f)

Figure 11. Trajectory planning model of sample a: (a) rank correlation of LSTM, (b) rank correlation of MLP, (c) rank correlation of Mul-Bi-LSTM, (d) $\mathrm{R}^{2}$ of LSTM, (e) $\mathrm{R}^{2}$ of MLP, (f) $\mathrm{R}^{2}$ of Mul-Bi-LSTM. 


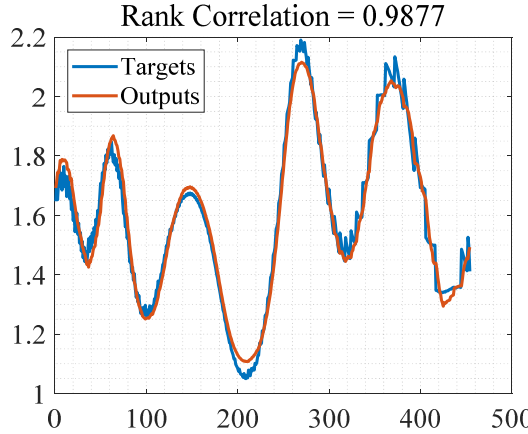

(a)

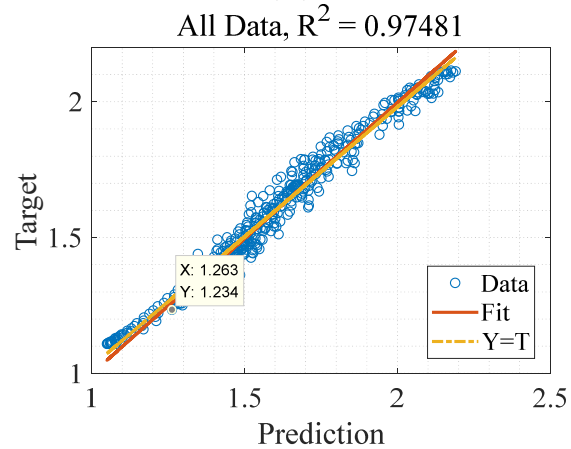

(d)

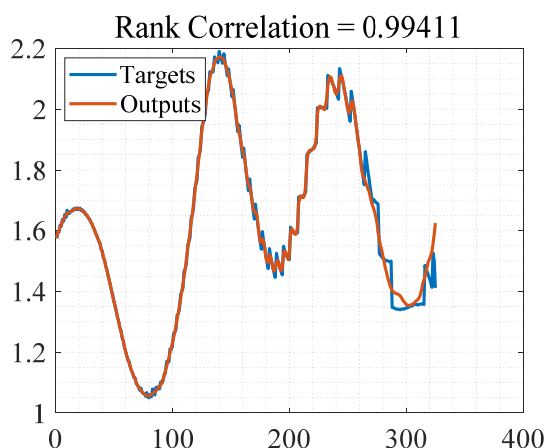

(b)

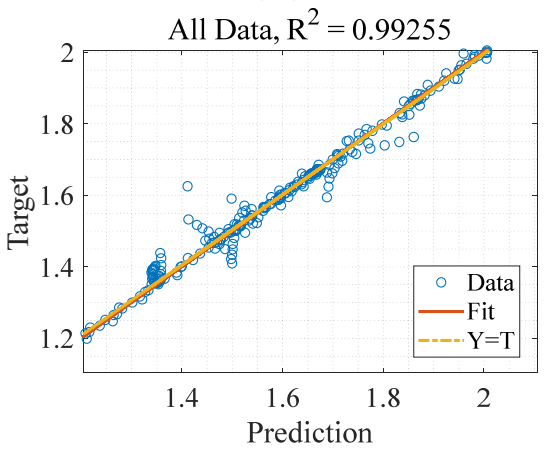

(e)

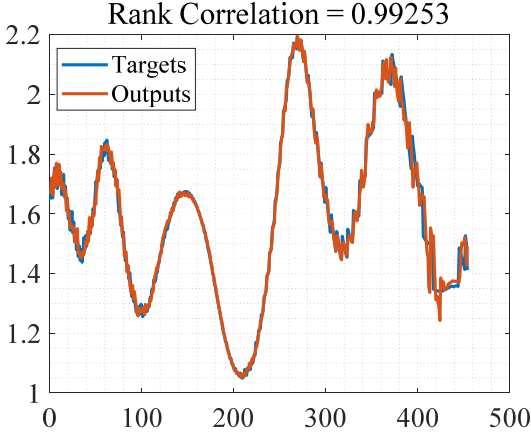

(c)

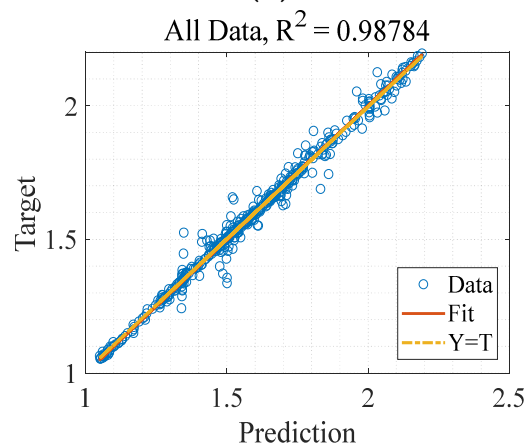

(f)

Figure 12. Trajectory planning model of sample b: (a) rank correlation of LSTM, (b) rank correlation of MLP, (c) rank correlation of Mul-Bi-LSTM, (d) $\mathrm{R}^{2}$ of LSTM, (e) $\mathrm{R}^{2}$ of MLP, (f) $\mathrm{R}^{2}$ of Mul-Bi-LSTM.

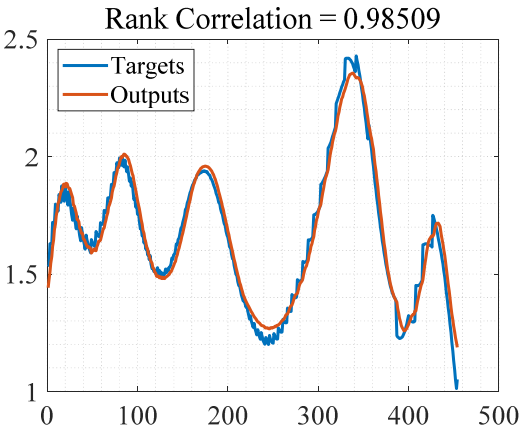

(a)

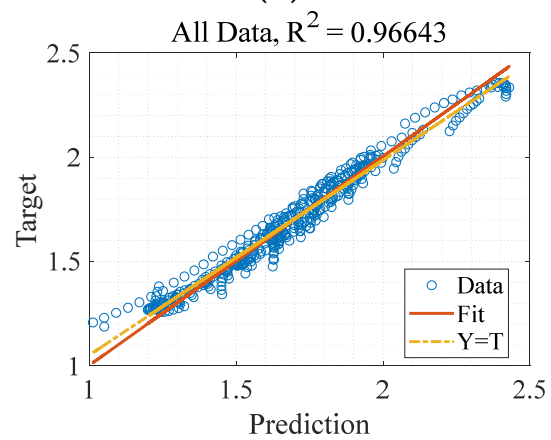

(d)

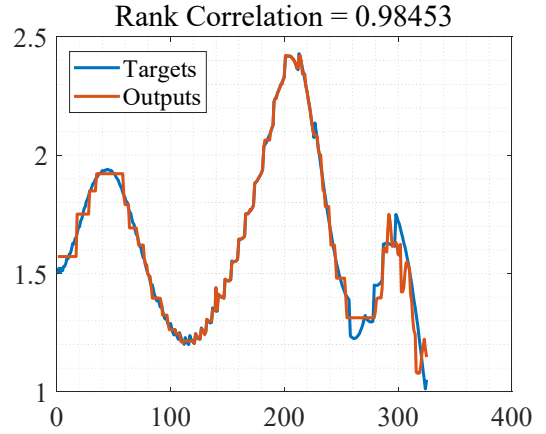

(b)

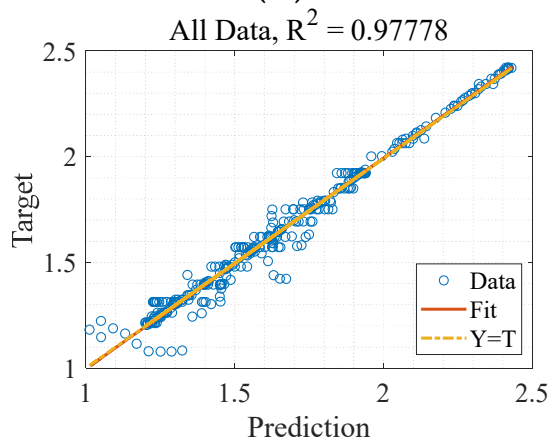

(e)

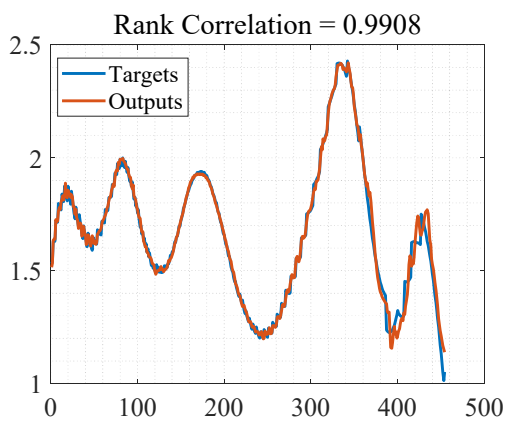

(c)

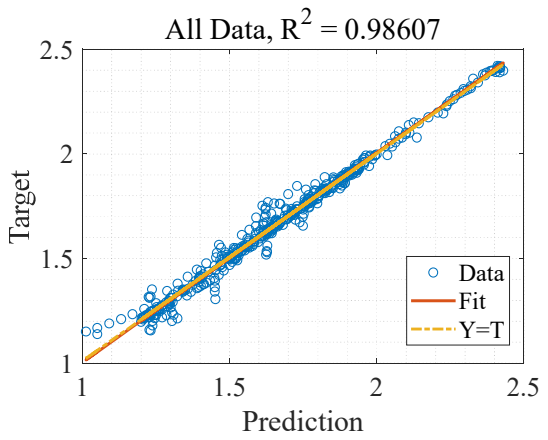

(f)

Figure 13. Trajectory planning model of sample c: (a) rank correlation of LSTM, (b) rank correlation of MLP, (c) rank correlation of Mul-Bi-LSTM, (d) $\mathrm{R}^{2}$ of LSTM, (e) R of MLP, (f) $\mathrm{R}^{2}$ of Mul-Bi-LSTM. 
As shown in Table 6, three test samples are randomly selected for model verification. In terms of Rank Correlation or $\mathrm{R}^{2}$, the prediction results of the Mul-Bi-LSTM trajectory planning model are generally better than those of LSTM and MLP. To further verify the applicability of the LSTM model framework between different curves, curves 6, 9 and 13 are randomly selected for performance validation, and all the sample data are summarized and analyzed to obtain the planned trajectory of each curve, as shown in Table 7 . It can be seen from Table 7 that in the curve trajectory planning model, the trajectory prediction value of Mul-Bi-LSTM is larger than that of LSTM. The rank correlation of Mul-Bi-LSTM trajectory prediction model in curve 6 increases by $1.3 \%$, and $\mathrm{R}^{2}$ increases by $11.65 \%$, compared with the LSTM model. The Rank correlation of Mul-Bi-LSTM trajectory prediction model in curve 9 increases by 1.49, and $\mathrm{R}^{2}$ increases by 14.35\%, compared with that of LSTM model. The rank correlation of Mul-Bi-LSTM trajectory prediction model in curve 13 increases by $1.64 \%$, and $\mathrm{R}^{2}$ increases by $4.85 \%$, compared with that of the LSTM model. Finally, in order to verify the portability of the LSTM model framework under the full sample condition, $80 \%$ of the samples are drawn as the training set and $20 \%$ of the samples are the test set. Mean square error (MSE), root mean squared error (RMSE) and normalized root mean squared error (NRMSE) are calculated to evaluate the prediction performance of the model. The results of each curve trajectory planning are shown in Table 8 . It can be seen from Table 8 that compared with LSTM and MLP, the mean error of Mul-Bi-LSTM is reduced by 0.00275 and 0.00742 , and the error Std is dropped by 0.00282 and 0.02955 , and the MSE is reduced by 0.00356 and 0.00261 . The results show that the value predicted by Mul-Bi-LSTM has a smaller deviation from the true value and is closer to the true value. Hence, the proposed Mul-Bi-LSTM possesses optimal prediction effect. With the introduction of human-like driving characteristic variables, the prediction performance of the model is significantly improved, and the prediction results are more accurate and closer to the true value, which is because there exists a strong recursive relationship between the trajectory data. The characteristics of driving variables can better describe and reflect the characteristics of the lateral offset, while the LSTM model separates the connections between the data, making its overall prediction performance lower, compared with that of the Mul-Bi-LSTM model.

Table 6. Comparison of samples.

\begin{tabular}{ccccccc}
\hline \multicolumn{4}{c}{ Rank Correlation } & \multicolumn{3}{c}{$R^{2}$} \\
\hline Sample & LSTM & MLP & Mul-Bi-LSTM & LSTM & MLP & Mul-Bi-LSTM \\
\hline Sample a & 0.9703 & 0.9734 & 0.9842 & 0.9380 & 0.9381 & 0.9637 \\
Sample b & 0.9877 & 0.9941 & 0.9925 & 0.9748 & 0.9926 & 0.9878 \\
Sample c & 0.9851 & 0.9845 & 0.9908 & 0.9664 & 0.9778 & 0.9861 \\
\hline
\end{tabular}

Table 7. Sample simulation results.

\begin{tabular}{ccccccc}
\hline & \multicolumn{3}{c}{ Rank Correlation } & \multicolumn{2}{c}{$\boldsymbol{R}^{\mathbf{2}}$} \\
\cline { 2 - 7 } & LSTM & Mul-Bi-LSTM & Contradistinction & LSTM & Mul-Bi-LSTM & Contradistinction \\
\hline Curve 6 & 0.9018 & 0.9148 & $1.3 \%$ & 0.7294 & 0.8459 & $11.65 \%$ \\
Curve 9 & 0.8897 & 0.9046 & $1.49 \%$ & 0.7216 & 0.8651 & $14.35 \%$ \\
Curve 13 & 0.9669 & 0.9833 & $1.64 \%$ & 0.9192 & 0.9677 & $4.85 \%$ \\
\hline
\end{tabular}

Table 8. Comparison of several models.

\begin{tabular}{cccccc}
\hline & Error Mean & Error Std & MSE & RMSE & NRMSE \\
\hline LSTM & 0.00393 & 0.05678 & 0.00416 & 0.06235 & 0.03876 \\
MLP & 0.00860 & 0.05396 & 0.00321 & 0.05612 & 0.03513 \\
Mul-Bi-LSTM & 0.00118 & 0.02441 & 0.00060 & 0.02441 & 0.01599 \\
\hline
\end{tabular}


From the previous results, it is clear that the Mul-Bi-LSTM model performed better than the MLP and LSTM models in terms of simulator data prediction. To further validate the adaptability of the Mul-Bi-LSTM model to the simulator data, a K-fold cross-validation method was used. This method evaluates the effect of the model, with the features that each sub-sample is involved in training and testing, and can reduce the generalisation error. $K$-fold cross-validation method is based on the idea that the data set is divided into $K$ regions. It selects different regions in turn as the test set. The remaining $K-1$ regions are used as the training set, and $K$ times of model validation are performed. The final average of the accuracy of the $K$ times validation results is taken as an estimate of the accuracy of the algorithm. The driving simulation result dataset was divided into 2 to 10 folds and the $R^{2}$ of each fold was estimated to prevent overfitting, as shown in the Figure 14 . It can be seen that the test results of six-fold cross-validation have the highest $R^{2}$. Combined with the results evaluated by the $K$-fold crossover method, the Bi-LSTM model proposed in this paper has good stability and generalisability.

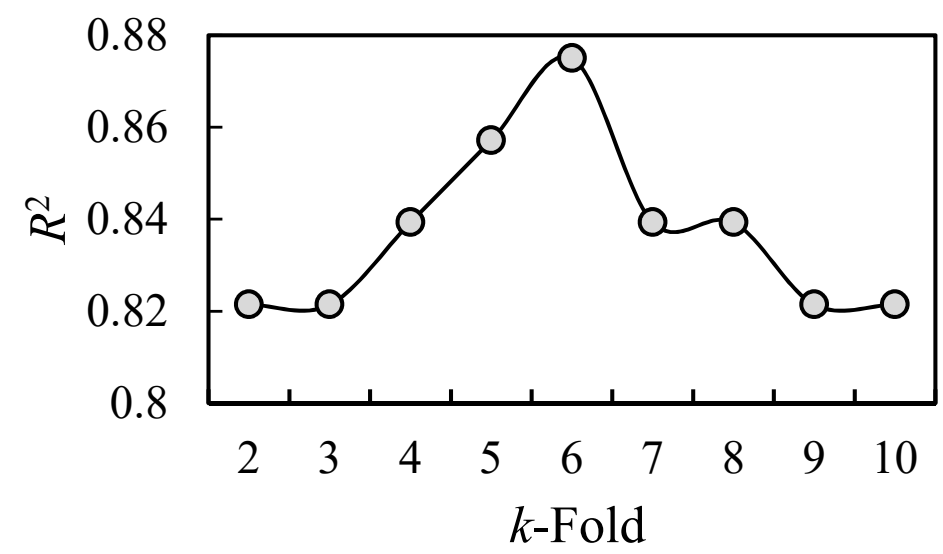

Figure 14. 2 to 10 -fold cross validation $R^{2}$.

\section{Conclusions}

This paper mainly evaluates the effectiveness of the driving behavior characteristics of the driver under the curved road conditions in the simulator. The analysis with the speed under curves and the curve vehicle trajectory are considered as the validation indicators. The driving simulator and 27 different types of testers are selected to design the curve experiment with the radii of $55 \mathrm{~m}, 150 \mathrm{~m}, 250 \mathrm{~m}, 350 \mathrm{~m}, 450 \mathrm{~m}$ and $550 \mathrm{~m}$ for validation of the experiment simulator. In the evaluation of vehicle speed, a method for evaluating vehicle speed consistency based on reliability coefficients is proposed, and the Kronbach's $\alpha$ reliability, split-half reliability and $r$ reliability are selected to conduct the reliability verification of the experimental speed results. Through sampling analysis, it is found that the overall reliability of the speed simulation for the tested driving simulator is relatively high, but the reliability of the speed simulation is low when turning in a small-radius curve. In the evaluation of speed accuracy of simulator curve, the speed values measured by the speed model at the starting point, midpoint and end point of the curve are adopted as calibration criteria, and the criterion-related validity, Cohen's $d$ exponent method is used for validity analysis. The results reveal that the test simulator perform preferable predictive efficiency in general.

In the evaluation of the curve trajectory, the concept of lateral offset is proposed as the virtual coordinate based on the longitudinal and lateral displacement of the vehicle with the designed road form pile number. The predicted trajectory obtained by the RRT model is adopted as the evaluation standard of the curve trajectory. In the comparison of indicators, the method of revised cosine trajectory similarity is selected for analysis. The experimental results show that the overall reliability of the vehicle turning trajectory is high when the curve radius is greater than or equal to $150 \mathrm{~m}$ in the test simulator, while the reliability of the vehicle turning trajectory is relatively poor when the curve radius is $55 \mathrm{~m}$. Based on the 
experimental results, in view of the low similarity of the curve trajectory between the RRT trajectory planning model and the simulator, the idea of using human-simulating operation data is proposed to establish a more complete human-simulating curve trajectory planning model by Mul-Bi-LSTM. Firstly, based on the trajectory similarity, it is concluded that the driver's curve trajectory presents strong regularity. Secondly, the trajectory planning performance of LSTM, MLP and Mul-Bi-LSTM models on the verification set and test set are compared. The results show that the Mul-Bi-LSTM model considering driving behavior factors can generate the most similar trajectory to the driver, which owns superior generalization performance. In conclusion, the speed and trajectory as the validation indicators, based on the velocity model and the Mul-Bi-LSTM trajectory model, can be used to evaluate the validity of the driving simulator under complex road conditions.

The future works to be investigated will include: (1) refinement of the validation system of the vehicle driving simulator, and (2) vehicle driving simulator development for intelligent transportation [51]. The validation of a driving simulator is a multi-level comprehensive system, which needs to be improved. The indicator system needs to be further refined, and the validation indicators need to be continuously modified through actual vehicle experiments. In addition, although the curve trajectory planning model based on human-simulating operation data driven by the thesis can be applied to emerging fields such as man-machine and autonomous driving, it is limited by experimental conditions. The effect in this process needs to be further developed for experimental scenarios and realization of related tests.

Author Contributions: Conceptualization, L.C. and F.G.; methodology, L.C.; investigation, L.C. and J.X.; data curation, W.T.; writing-original draft preparation, L.C. and S.W.; writing-review and editing, F.G. and Z.C.; supervision, F.G.; funding acquisition, F.G. All authors have read and agreed to the published version of the manuscript.

Funding: This research was funded by NATIONAL NATURAL SCIENCE FOUNDATION OF CHINA, Grant Number 71961012.

Informed Consent Statement: Informed consent was obtained from all subjects involved in the study.

Data Availability Statement: Not applicable.

Conflicts of Interest: The authors declare no conflict of interest.

\section{Appendix A}

Table A1. Detailed information of drivers.

\begin{tabular}{cccccc}
\hline No. & Sex & $\begin{array}{c}\text { Driving } \\
\text { Years/Kilo Miles }\end{array}$ & Age & $\begin{array}{c}\text { Accidents in } \\
\text { Recent Three } \\
\text { Years (Y/N) }\end{array}$ & $\begin{array}{c}\text { Violation in } \\
\text { Recent Three } \\
\text { Years (Y/N) }\end{array}$ \\
\hline 1 & Female & 9 years $/ 50-60$ & 35 & $\mathrm{~N}$ & $\mathrm{~N}$ \\
2 & Male & 24 years $/ 250$ & 56 & $\mathrm{~N}$ & $\mathrm{Y}$ \\
3 & Male & 9 years $/ 100$ & 38 & $\mathrm{~N}$ & $\mathrm{Y}$ \\
4 & Male & 9 years $/ 100$ & 38 & $\mathrm{~N}$ & $\mathrm{~N}$ \\
5 & Male & 10 years $/ 80$ & 43 & $\mathrm{~N}$ & $\mathrm{Y}$ \\
6 & Male & 4 years $/ 30$ & 28 & $\mathrm{~N}$ & $\mathrm{Y}$ \\
7 & Male & 8 years $/ 140$ & 41 & $\mathrm{Y}$ & $\mathrm{N}$ \\
8 & Female & 7 years $/ 10$ & 41 & $\mathrm{~N}$ & $\mathrm{~N}$ \\
9 & Female & 10 years $/ 50$ & 38 & $\mathrm{~N}$ & $\mathrm{Y}$ \\
10 & Male & 24 years $/ 200$ & 46 & $\mathrm{~N}$ & $\mathrm{Y}$ \\
11 & Female & 8 years $/ 70$ & 39 & $\mathrm{~N}$ & $\mathrm{Y}$ \\
12 & Female & 6 years $/ 50$ & 48 & $\mathrm{~N}$ & $\mathrm{~N}$ \\
13 & Female & 12 years $/ 100$ & 39 & & \\
\hline
\end{tabular}


Table A1. Cont.

\begin{tabular}{llcccc}
\hline No. & Sex & $\begin{array}{c}\text { Driving } \\
\text { Years/Kilo Miles }\end{array}$ & Age & $\begin{array}{c}\text { Accidents in } \\
\text { Recent Three } \\
\text { Years (Y/N) }\end{array}$ & $\begin{array}{c}\text { Violation in } \\
\text { Recent Three } \\
\text { Years (Y/N) }\end{array}$ \\
\hline 14 & Female & 5 years $/ 50$ & 32 & $\mathrm{~N}$ & $\mathrm{~N}$ \\
15 & Female & 14 years $/ 60$ & 33 & $\mathrm{~N}$ & Y \\
16 & Female & 14 years $/ 60$ & 38 & $\mathrm{~N}$ & $\mathrm{Y}$ \\
17 & Male & 15 years $/ 150$ & 37 & $\mathrm{~N}$ & $\mathrm{Y}$ \\
18 & Male & 5 years $/ 60$ & 24 & $\mathrm{~N}$ & $\mathrm{~N}$ \\
19 & Male & 4 years $/ 100$ & 23 & $\mathrm{~N}$ & $\mathrm{~N}$ \\
20 & Male & 3 years $/ 10$ & 23 & $\mathrm{~N}$ & $\mathrm{~N}$ \\
21 & Male & 20 years $/ 300$ & 51 & $\mathrm{~N}$ & $\mathrm{~N}$ \\
22 & Male & 3 years $/ 50$ & 33 & $\mathrm{~N}$ & $\mathrm{~N}$ \\
23 & Male & 4 years $/ 50$ & 23 & $\mathrm{~N}$ & $\mathrm{~N}$ \\
24 & Male & 3 years $/ 20$ & 24 & $\mathrm{~N}$ & $\mathrm{~N}$ \\
25 & Male & 3 years $/ 20$ & 24 & $\mathrm{~N}$ & $\mathrm{~N}$ \\
26 & Male & 4 years $/ 80$ & 22 & $\mathrm{~N}$ & $\mathrm{~N}$ \\
27 & Male & 3 years $/ 20$ & 21 & & $\mathrm{~N}$ \\
\hline
\end{tabular}

\section{References}

1. Wynne, R.A.; Beanland, V.; Salmon, P.M. Systematic review of driving simulator validation studies. Saf. Sci. 2019, 117, 138-151. [CrossRef]

2. Xiong, J.; Zeng, J.G.; Ding, L.; Chen, Z.L.; Shui, R.F. Application and research of vehicle driving simulator for road traffic problems. China J. Highw. Transp. 2002, 15, 117-119.

3. Broughton, K.L.M.; Switzer, F.; Scott, D. Car following decisions under three visibility conditions and two speeds tested with a driving simulator. Accid. Anal. Prev. 2007, 39, 106-116. [CrossRef] [PubMed]

4. Cox, S.M.; Cox, D.J.; Kofler, M.J. Driving Simulator Performance in Novice Drivers with Autism Spectrum Disorder: The Role of Executive Functions and Basic Motor Skills. J. Autism Dev. Disord. 2016, 46, 1379-1391. [CrossRef] [PubMed]

5. Xiong, J.; Wu, A.; Qin, Y. Experimental study on the effectiveness of driving simulator training. Chin. J. Ergon. $2016,22,63-67$.

6. Caffò, A.O.; Tinella, L.; Lopez, A.; Spano, G.; Massaro, Y.; Lisi, A.; Stasolla, F.; Catanesi, R.; Nardulli, F.; Grattagliano, I.; et al. The Drives for Driving Simulation: A Scientometric Analysis and a Selective Review of Reviews on Simulated Driving Research. Front. Psychol. 2020, 11, 917. [CrossRef] [PubMed]

7. Bham, G.H.; Leu, M.C.; Vallati, M.; Mathur, D.R. Driving simulator validation of driver behavior with limited safe vantage points for data collection in work zones. J. Saf. Res. 2014, 49, 53-60. [CrossRef]

8. Meuleners, L.; Fraser, M. A validation study of driving errors using a driving simulator. Transp. Res. Part F Traffic Psychol. Behav. 2015, 29, 14-21. [CrossRef]

9. Dong, L.; Sun, D.; Han, G.; Li, X.; Hu, Q.; Shu, L. Velocity-free Localization of Autonomous Driverless Vehicles in Underground Intelligent Mines. IEEE Trans. Veh. Technol. 2020, 69, 9292-9303. [CrossRef]

10. Chen, X.-M.; Miao, Y.S. Driving decision-making analysis of car-following for autonomous vehicle under complex urban environment. J. Cent. South Univ. 2017, 24, 1476-1482. [CrossRef]

11. Cao, C.; Luo, Y.; Wang, J. Driving simulator validation for research on driving behavior at entrance of urban underground road. In Proceedings of the 2015 International Conference on Transportation Information and Safety, Wuhan, China, 25-28 June 2015; pp. 147-150.

12. Bella, F. Driver perception hypothesis: Driving simulator study. Transp. Res. Part F Traffic Psychol. Behav. 2014, 24, 183-196. [CrossRef]

13. Heiman, S.; Reed, N. Validation of the driver behaviour questionnaire using behavioural data from an instrumented vehicle and high-fidelity driving simulator. Accid. Anal. Prev. 2015, 75, 245-251.

14. Tozman, T.; Magdas, E.S.; MacDougall, H.G.; Vollmeyer, R. Understanding the psychophysiology of flow: A driving simulator experiment to investigate the relationship between flow and heart rate variability. Comput. Hum. Behav. 2015, 52, 408-418. [CrossRef]

15. Jesse, M.; Romain, C.; David, N.T. Driving simulator scenarios and measures to faithfully evaluate risky driving behavior: A comparative study of different driver age groups. PLOS ONE 2017, 12, e0185909.

16. Li, X.; Wang, W.; Roetting, M. Estimating Driver's Lane-Change Intent Considering Driving Style and Contextual Traffic. IEEE Trans. Intell. Transp. Syst. 2018, 20, 3258-3271. [CrossRef]

17. Zheng, R.; Nakano, K.; Yamabe, S.; Aki, M.; Nalkamura, H.; Suda, Y. Study on Emergency-Avoidance Braking for the Automatic Platooning of Trucks. IEEE Trans. Intell. Transp. Syst. 2014, 15, 1748-1757. [CrossRef]

18. Gemou, M. Transferability of driver speed and lateral deviation measurable performance from semi-dynamic driving simulator to real traffic conditions. Eur. Transp. Res. Rev. 2013, 5, 217-233. [CrossRef] 
19. Groeger, J.A.; Murphy, G. Driver performance under simulated and actual driving conditions: Validity and orthogonality. Accid. Anal. Prev. 2020, 143, 105593. [CrossRef] [PubMed]

20. Zhang, Y.; Guo, Z.; Sun, Z. Driving Simulator Validity of Driving Behavior in Work Zones. J. Adv. Transp. 2020, $2020,4629132$. [CrossRef]

21. Zhou, X.; Jiang, H.; Li, A.; Ma, S. A New Single Point Preview-Based Human-Like Driver Model on Urban Curved Roads. IEEE Access 2020, 8, 107452-107464. [CrossRef]

22. Dixit, S.; Fallah, S.; Montanaro, U.; Dianati, M.; Stetvens, A.; McCullough, F.; Mouzakitis, A. Trajectory planning and tracking for autonomous overtaking: State-of-the-art and future prospects. Annu. Rev. Control 2018, 45, 76-86. [CrossRef]

23. Haigen, G. Psychology and Educational Measurement; Peking University Press: Beijing, China, 2008; pp. 34-40.

24. Jin, X.; Jia, Z.; Xu, W.; Yi, S. Longitudinal Acceleration Performance of Passenger Cars on Complex Mountain Highways. China J. Highw. Transp. 2017, 30, 115-126.

25. Sawada, T.; Tomori, K.; Hamana, H.; Ohno, K.; Seike, Y.; Igari, Y.; Fujita, Y. Reliability and validity of on-road driving tests in vulnerable adults: A systematic review. Int. J. Rehabil. Res. 2019, 42, 289-299. [CrossRef] [PubMed]

26. Jiang, H.; Zhou, J.; Li, A.; Zhou, X.; Ma, S. Human-like trapezoidal steering angle model on two-lane urban curves. Int. J. Adv. Robot. Syst. 2019, 16, 1729881419867614. [CrossRef]

27. Noreen, I.; Khan, A.; Habib, Z. Optimal Path Planning using RRT based Approaches: A Survey and Future Directions. Int. J. Adv. Comput. Sci. Appl. (IJACSA) 2016, 7, 142-146. [CrossRef]

28. Taylor, J.; Zhou, X.; Rouphail, N.M.; Portetr, R.J. Method for investigating intradriver heterogeneity using vehicle trajectory data: A Dynamic Time Warping approach. Transp. Res. Part B 2015, 73, 59-80. [CrossRef]

29. Knauss, A.; Berger, C.; Eriksson, H. Proving Ground Support for Automation of Testing of Active Safety Systems and Automated Vehicles. In Proceedings of the Fourth Internetional Symposium on Future Active Safety Technology toward Zero Traffic Accidents (FASTzero), Nara, Japan, 18-21 September 2017.

30. Zhang, Y.; Xiong, R.; He, H. Long Short-Term Memory Recurrent Neural Network for Remaining Useful Life Prediction of Lithium-Ion Batteries. IEEE Trans. Veh. Technol. 2018, 67, 5695-5705. [CrossRef]

31. Xiong, R.; Cao, J.; Quanqing, Y. Reinforcement learning-based real-time power management for hybrid energy storage system in the plug-in hybrid electric vehicle. Appl. Energy 2018, 211, 538-548. [CrossRef]

32. Zhang, Y.; Huang, Y.; Chen, Z.; Li, G.; Liu, Y. A Novel Learning Based Model Predictive Control Strategy for Plug-in Hybrid Electric Vehicle. IEEE Trans. Transp. Electrif. 2021. [CrossRef]

33. Liu, Y.; Zhao, P.; Qin, D.; Yang, Y.; Chen, Z. Driving Intention Identification Based on Long Short-Term Memory Neural Network. In Proceedings of the 2019 IEEE Vehicle Power and Propulsion Conference (VPPC), Hanoi, Vietnam, 14-17 October 2019.

34. Chen, Z.; Zhao, H.; Shu, X. Synthetic state of charge estimation for lithium-ion batteries based on long short-term memory network modeling and adaptive H-Infinity filter. Energy 2021, 228, 120630. [CrossRef]

35. Li, A.; Jiang, H.; Li, Z.; Zhou, J.; Zhou, X. Human-Like Trajectory Planning on Curved Road: Learning From Human Drivers. IEEE Trans. Intell. Transp. Syst. 2019, 21, 3388-3397. [CrossRef]

36. Li, X.; Yan, X.; Wong, S.C. Effects of fog, driver experience and gender on driving behavior on S-curved road segments. Accid. Anal. Prev. 2015, 77, 91-104. [CrossRef] [PubMed]

37. Scialfa, C.T.; Borkenhagen, D.; Lyon, J.; Deschênes, M.; Horswill, M.; Wetton, M. The effects of driving experience on responses to a static hazard perception test. Accid. Anal. Prev. 2012, 45, 547-553. [CrossRef]

38. Design Specification for Highway Alignment; JTG D20-2017; Ministry of Communications of the People's Republic of China: Beijing, China, 2017.

39. Zeng, J.; Xiong, J.; Wan, H. Development and Application for 3D Scene Generated System of Driving Simulator. J. Syst. Simul. 2002, 14, 752-755.

40. Qin, Y.; Xiong, J.; Jiang, Y.; Guo, F.; Wan, H.; Jiang, L.; Jia, X. Simulator Evaluation of Drivers' Performance on Rural Highways in relation to Drivers' Visual Attention Demands. Adv. Mech. Eng. 2015, 7, 249257. [CrossRef]

41. Xie, X. $\gamma$ coefficient as an estimation of reliability. J. Chin. Psychol. Acta Psychol. Sin. 1998, 30, $193-196$.

42. Xie, X. Comparison of 15 equating methods. J. Chin. Psychol. Acta Psychol. Sin. 2000, 32, 217-222.

43. Li, H. The Confirmation of Measurability: Epistemological Foundation of Psychological and Educational Measurement. Psychol. Explor. 2015, 304-306.

44. Huajie Engineering Consulting Co., Ltd. Specifications for Highway Safety Audit; JTG B05-2015; People's Communications Press Co., Ltd.: Beijing, China, 2016.

45. Kuffner, J., Jr.; Lavalle, S.M. RRT-Connect: An Efficient Approach to Single-Query Path Planning. In Proceedings of the 2000 IEEE International Conference on Robotics and Automation, San Francisco, CA, USA, 24-28 April 2000.

46. Chen, J.; Chen, Y.; Tian, F. The Method of Sample Generation for Power Grid Simulation Based on LSTM. Proc. CSEE 2019, 39, 4129-4134.

47. Alkhwiter, W.; Al-Twairesh, N. Part-of-speech tagging for Arabic tweets using CRF and Bi-LSTM. Comput. Speech Lang. 2020, 65, 101138. [CrossRef]

48. Rosenblatt, F. The perceptron: A probabilistic model for information storage and organization in the brain. Psychol. Rev. 1958, 65, 386-408. [CrossRef] [PubMed] 
49. Mathematical Statistics Group, Institute of Mathematics, Chinese Academy of Sciences. Regression Analysis Method; Science Press: Beijing, China, 1974.

50. Li, T.; Ni, A.; Zhang, C. Short-term traffic congestion prediction with Conv-BiLSTM considering spatio-temporal features. IET Intell. Transp. Syst. 2021, 14, 1978-1986. [CrossRef]

51. Seif, H.G.; Hu, X. Autonomous Driving in the iCity-HD Maps as a Key Challenge of the Automotive Industry. Engineering 2016, 2, 159-162. [CrossRef] 